\title{
A multi-field model for charging and discharging of lithium-ion battery electrodes
}

Received: 29 June 2020 / Accepted: 15 October 2020 / Published online: 6 November 2020

(c) The Author(s) 2020

\begin{abstract}
An electrochemical-thermomechanical model for the description of charging and discharging processes in lithium electrodes is presented. Multi-physics coupling is achieved through the constitutive relations, obtained within a consistent thermodynamic framework based on the definition of the free energy density, sum of distinct contributions from different physics. The system is characterized by finite kinematics, under the assumption of locality of deformation, and the deformation gradient is decomposed into the product of elastic and inelastic parts. Specifically, a Taylor series expansion is used to approximate the inelastic deformation due to ion intercalation. The elastic part can be described alternatively by two finite kinematics models of neo-Hookean elasticity, and a Maxwell-type viscoelastic model accounts for time-dependent mechanical aspects. The model is implemented into a finite element code that uses B-spline basis functions. We illustrate the features of the model by means of selects examples, showing that chemo-mechanical interaction affects the equilibrium concentrations of the phases. The model captures the fundamental aspects of the anode charging and discharging processes.
\end{abstract}

Keywords Lithium batteries - Coupled processes · Thermomechanics · Electrochemistry · Viscosity · Finite kinematics · Finite elements

\section{Introduction}

The increasing use of portable electronics, handy devices, and electric automotive conveys an impending growth in the demand for secondary batteries [1], among which the most successful at the present are lithium based. In the automotive industry alone, the world market for lithium-based batteries could grow from the registered $\$ 24$ billion in 2017 to $\$ 65$ billion by 2025 [53]. This extraordinary potential explains the strong interest in the development of high energy-density batteries with optimized electrodes for which design intuition, experimental results, and numerical simulations are needed.

Communicated by Andreas Öchsner.

M. Werner $(\varangle) \cdot \mathrm{K}$. Weinberg

Fakultät IV, Department Maschinenbau, Lehrstuhl für Festkörpermechanik, Universität Siegen, Paul-Bonatz-Str. 9-11, 57076 Siegen, Germany

E-mail: marek.werner@uni-siegen.de

K. Weinberg

E-mail: kerstin.weinberg@uni-siegen.de

A. Pandolfi

Dipartimento di Ingegneria Civile ed Ambientale, Politecnico Milano, Piazza Leonardo da Vinci 32, 20133 Milan, Italy

E-mail: anna.pandolfi@polimi.it 
Making use of the classical Boltzmann continuum assumptions [50], this study describes a multi-physics numerical model able to simulate the electrochemical process of charging and discharging of an electrode. The coupled constitutive relations are obtained through the thermodynamical approach of Coleman and Noll [12], based on the assumption that the local state of a system is entirely described by the deformation, the entropy, and a set of suitable internal variables defined within a small neighborhood of a material point. The proposed model extends previous models by $[2,8,22,39,65]$ and others, by coupling the full electrochemical-thermomechanical process of charging and discharging with large reversible and irreversible mechanical deformations.

In general, the performance of the electrodes during charge-induced ionic flow is size, geometry, and structure dependent $[7,19,29,58]$. The bulk material may be subjected to structural changes, e.g., a transformation from crystalline to amorphous has been reported for silicon anodes [23,36]. Geometrical aspects coupled with the electrode design have been investigated experimentally, revealing that hollow micron-sized silicon structures are advantageous high-performance particles [64] and that solid amorphous silicon rods do not crack [10]. In common commercial electrode materials, the lithium ion content induces material softening or hardeningan effect which has been observed experimentally [41] and predicted in first principle density functional theory computations $[37,42]$. Moreover, the formulation of high-fidelity models for specific electrode compositions is an active area of research, see $[8,17,31]$ among others.

Most approaches used to model batteries account for small deformations and linear elasticity, cf. [8,22,57]. The description is comparatively easy and is adequate for many applications, but new developments, such as silicon anodes, require to account for considerable changes of shape and volume. A simple coupled largedeformation model for anode charging, neglecting chemical decomposition and phase separation, was proposed in [13]. A continuum-level theory, coupling diffusion and finite deformations, was proposed in [2] and later solved in a simplified manner by standard finite elements (FEM), [26,38]. Typical cathode materials, such as lithium manganate, have been investigated for stress-related phase separation $[22,66]$ also using a nonlocal diffusion model [62]. Advanced FEM simulations indicated that the formation of a core-shell structure able to inhibit further lithiation depends strongly on the choice of kinetic parameters and on the state of charge [67]. Cracking of the electrode material has also been investigated in [47], although it has not been observed experimentally [10].

In abstract terms, charging and discharging of a lithium-ion battery electrode result from particle exchange between the anode material A (e. g., silicon or graphite) and the electrolyte (e. g., LiPF6 salt),

$$
\mathrm{A}+\mathrm{e}^{-}+\mathrm{Li}^{+} \rightleftharpoons \mathrm{ALi} .
$$

The physical processes that affect the electrochemical reaction at the electrode are: ion flux and diffusion, electrical and thermal conduction, swelling and mechanical deformations, and electro-mechanical forces associated with the electrostatic field. The corresponding field equations are illustrated in Sect. 2. The thermodynamical consistent derivation of the general constitutive equations for the coupled electrochemical-thermomechanical process of charging and discharging is outlined in Sect. 3. In Sect. 4, we provide the constitutive model for lithium electrodes by defining the specific Helmholtz free energy density and using its partial derivatives to obtain the mechanical stresses, the thermal entropy, the electric induction, and the chemical potential of the $\mathrm{Li}^{+}$enriched electrode material. The properties of the coupled model are illustrated in Sect. 5, comparing the proposed approach with other models from literature. In Sect. 6, we provide FEM examples demonstrating the interactions of the different physical fields. The features of the proposed electrochemical-thermomechanical model are summarized in Sect. 7, while Appendix provides some fundamental relations.

\section{Field equations}

A point of a continuum in its material configuration $\mathcal{B}_{0} \subset \mathbb{R}^{3}$ is denoted by $\boldsymbol{X}$. An arbitrary deformation in a time interval $\left[0, t_{\text {end }}\right]$ moves the material point to a new position $\boldsymbol{x}(\boldsymbol{X}, t)$ in the current configuration $\mathcal{B}_{t} \subset \mathbb{R}^{3}$. The corresponding deformation mapping $\chi(X, t): \mathcal{B}_{0} \times\left[0, t_{\text {end }}\right] \rightarrow \mathcal{B}_{t}$ is uniquely described by its material gradient,

$$
\boldsymbol{F}(\boldsymbol{X}, t)=\frac{\partial \chi(\boldsymbol{X}, t)}{\partial \boldsymbol{X}}=\nabla_{\boldsymbol{X}} \boldsymbol{x}(\boldsymbol{X}, t) .
$$

The local volume change at $\boldsymbol{x}$ is measured by the Jacobian determinant $J=\operatorname{det} \boldsymbol{F}$, which assigns an infinitesimal volume element $\mathrm{d} V$ of its material configuration to its spatial configuration $\mathrm{d} v, \mathrm{~d} v=J \mathrm{~d} V$. 
Table 1 Field equations for the electrochemical-thermomechanical multi-field problem: $\rho$-density, $h$-mass rate density, $h_{k}$ mass rate density of component $k, c_{k}$-concentration of component $k, j$-mass flux vector, $v_{i j}$-stoichiometric coefficient, $\mathcal{J}_{j}$-reaction rate, $\boldsymbol{\sigma}$-Cauchy stress, $\boldsymbol{b}$-body force density, $\boldsymbol{v}$ - velocity, $\boldsymbol{d}$ - electrical induction, $\rho_{e} z$ - charge density, $C_{T}-$ heat capacity per unit volume, $\boldsymbol{h}_{T}$-heat flux vector

\begin{tabular}{lll}
\hline Total mass balance & $\dot{\rho}+\rho \dot{J} / J=h$ & \\
Mass flux & $h=\sum_{k} h_{k}$ & (2) \\
Diffusion/reaction & $\rho \dot{c}_{k}=\left(h_{k}-c_{k} h\right)-\operatorname{div} \boldsymbol{j}+\Sigma_{j} \mathcal{J}_{j} v_{k j}$ & $(4)$ \\
Linear momentum balance & $\operatorname{div} \boldsymbol{\sigma}+\boldsymbol{b}=\rho \dot{\boldsymbol{v}}$ & $(5)$ \\
Angular momentum balance & $\boldsymbol{\sigma}^{\top}=\boldsymbol{\sigma}$ & (6) \\
Electrical induction & $\operatorname{div} \boldsymbol{d}=\rho_{e} z$ & (7) \\
Thermal diffusion & $C_{T} \dot{T}=\mathcal{R}+\operatorname{div} \boldsymbol{h}_{T}$
\end{tabular}

The field equations that govern the mass, concentration, elastic, electrostatic, and thermal fields are summarized in Table 1 in the common spatial form. Corresponding quantities of the material configuration will be written with capital letters. The complete notation is listed in Table 3 of Appendix, where also the transformation rules between the configurations are provided. In the following, we illustrate the equations of each of the physical processes in turn.

\subsection{Partial and total mass balance}

We consider a general multi-component system consisting of $n$ species. The mass density $\rho_{k}(\boldsymbol{x}, t)$ of the $k$-th species represents the mass $m_{k}(\boldsymbol{x}, t)$ per current volume $v$, and the sum gives the total mass density $\rho$

$$
\rho_{k}(\boldsymbol{x}, t)=\frac{\mathrm{d} m_{k}}{\mathrm{~d} v} \text { and } \rho(\boldsymbol{x}, t)=\sum_{k=1}^{n} \rho_{k} .
$$

The electrode charging and discharging implies mass transfer, described by a rate per unit of current volume $h$. The evolution of the local density field follows from Eq. (2), which provides the conservation of the mass $J=\rho_{0} / \rho$ in the special case of $h=0$.

\subsection{Concentration and particle diffusion}

The mass fraction of the $k$-th component $\rho_{k}$ is defined as

$$
c_{k}=\frac{\rho_{k}}{\rho} \in[0,1]
$$

and is further referred to as concentration. By definition, it holds $\sum_{k=1}^{n} c_{k}=1$. The global mass balance for each of the $n$ individual species can be formulated by introducing the inward mass flux $j_{k}=-\boldsymbol{j}_{k} \cdot \boldsymbol{n}$, where $\boldsymbol{n}$ is the unit vector normal to the boundary. The total mass change in Eq. (3) accounts for the single component mass transfer through the density rate $h_{k}$, induced by the chemical reactions occurring inside the body (see below). According to the localization theorem (see Appendix, (66)), the total mass rate contributes to the local mass flux, Eq. (4), through the concentration $c_{k}(10)$.

The diffusive fluxes are assumed to depend on the spatial gradient of the molar chemical potential $\mu_{k}$ through a second-order tensor of particle mobilities $\boldsymbol{m}_{i k}$,

$$
\boldsymbol{j}_{k}=-\sum_{i=1}^{n} \boldsymbol{m}_{i k}\left(c_{i}, T\right) \nabla_{\boldsymbol{x}} \mu_{i} .
$$

The molar chemical potential $\mu_{k}$ comprises the free energy gain for adding one mole of $k$-th component to the system and does not depend on the configuration. For the special case of mass conservation, under the 
assumptions of isotropic mobility, absence of chemical reactions, and constituents of approximately equal molar mass, the temporal evolution of the concentration simplifies to

$$
\rho \dot{c}_{k}=\nabla_{\boldsymbol{x}} \cdot\left(\sum_{i=1}^{n} m_{i k} \nabla_{\boldsymbol{x}} \mu_{i}\right)
$$

where $m_{i k}=\rho D_{i k} c_{i} c_{k} / \theta(T)$ is the degenerate mobility and $\boldsymbol{m}_{i k}=m_{i k} \mathbb{1}$. The temperature dependent diffusivity $D_{i j}=D_{i j}(T)$ is typically modeled with the Arrhenius law. A straightforward calculation using the relations of the Appendix shows that Eq. (12) can be expressed in the same way with the material gradient $\nabla_{X} \mu$.

\subsection{Chemical reactions}

In addition to the pure diffusion of the species, we consider here a typical binary chemical reaction

$$
n_{A} A+n_{B} B \stackrel{\mathcal{J}}{\rightarrow} n_{C} C+n_{D} D
$$

where the reactant consists of $n_{A}$ and $n_{B}$ moles of molecules of the species $A$ and $B$, respectively, which react to produce $n_{C}$ and $n_{D}$ moles of molecules of species $C$ and $D$, respectively. The reaction rate $\mathcal{J}$ of the chemical reaction gives information on the kinetics of the chemical reaction. Generally, we decompose the reaction into $r$ stoichiometrically independent reaction pathways with rates $\mathcal{J}_{j}, j \in\{1,2, \ldots, r\}$. The driving forces of the process, i. e., the reaction affinities $\mathcal{A}_{j}$, are assumed to derive from a variational principle where the chemical species are subject to constant supply rates [18]. The chemical affinity $\mathcal{A}_{j}$ of the $j$-th reaction is then defined as

$$
\mathcal{A}_{j}=\sum_{k=1}^{n} v_{k j} \mu_{k}, \quad j \in\{1,2, \ldots, r\} .
$$

This contributes to the local mass evolution of the $k$-th component (last term in Eq. (4)). Since mass is conserved in each separate chemical reaction, it holds $\sum_{k=1}^{r} v_{k j}=0$.

\subsection{Linear and angular momentum balance}

The deformation of a system follows Newton's laws of motion. For an overall Cauchy stress field $\boldsymbol{\sigma}(\boldsymbol{x}, t)$, a mean velocity $\boldsymbol{v}(\boldsymbol{x}, t)$ and a body force per unit volume $\boldsymbol{b}$, Newton's law reduces to the local form of the linear momentum balance (5) and of the angular momentum balance (6). A surface traction $\boldsymbol{t}=\boldsymbol{\sigma} \cdot \boldsymbol{n}$ may be assigned at the boundary. Here, the dynamics of $n$-component mixtures, i.e., convective effects and inertia of the different components, will be neglected, cf. [3].

For the material description, we employ the first Piola-Kirchhoff stress tensor $\boldsymbol{P}$

$$
\boldsymbol{P}=J \boldsymbol{\sigma} \boldsymbol{F}^{-\top} .
$$

The angular momentum balance is then satisfied through the symmetry of the product $\boldsymbol{P} \boldsymbol{F}^{\top}=\boldsymbol{F} \boldsymbol{P}^{\top}$.

\subsection{Electrostatics}

The spatial electric field $\boldsymbol{e}$ is given by the spatial gradient of an electric potential $\phi_{e}, \boldsymbol{e}(\boldsymbol{x}, t)=-\nabla_{\boldsymbol{x}} \phi_{e}(\boldsymbol{x}, t)$, and the electric induction (or electric displacement) $\boldsymbol{d}$ is proportional to the electric field through the vacuum permittivity $\varepsilon_{0}$. For a dielectric material, a common constitutive relation assumes $\boldsymbol{d}=\varepsilon_{0} \varepsilon_{r} \boldsymbol{e}$, where $\varepsilon_{r}$ is a concentration and temperature-dependent relative permittivity. For brevity, we write $\varepsilon_{e}(c, T)=\varepsilon_{0} \varepsilon_{r}(c, T)$.

The governing equation for electrostatics is $\nabla_{\boldsymbol{x}} \times \boldsymbol{e}=\mathbf{0}$ and, for a local charge density $\rho_{e} z$, the electric induction must satisfy the equilibrium Eq. (7). Here, the mixture current fluxes between components are neglected, cf. [56].

For later reference, we formulate the electrostatic relations with the corresponding material fields, $\boldsymbol{E}=\boldsymbol{F}^{\top} \boldsymbol{e}$ and $\boldsymbol{D}=J \boldsymbol{F}^{-1} \boldsymbol{d}$. Then, the material electric induction follows as

$$
\boldsymbol{D}=J \varepsilon_{e}\left(\mathbf{F}^{\top} \mathbf{F}\right)^{-1} \boldsymbol{E} .
$$




\subsection{Heat conduction}

Heat transfer is assumed to be governed by Fourier's Eq. (8). The material thermal flux $\boldsymbol{H}_{T}$ relates to its spatial counterpart $\boldsymbol{h}_{T}$ by $\boldsymbol{H}_{T}=J \boldsymbol{F}^{-1} \boldsymbol{h}_{T}$. Commonly the spatial thermal flux is assumed to be linearly dependent on the gradient of the temperature through a spatial second-order tensor of thermal conductivities $\boldsymbol{k}_{T}$. The constitutive relation in material form follows through a pull-back of the thermal conductivities, $\boldsymbol{K}_{T}=\boldsymbol{F}^{-1} \boldsymbol{k}_{T} \boldsymbol{F}^{-\top}$, see Appendix 7, as

$$
\boldsymbol{H}_{T}=-\boldsymbol{K}_{T} \nabla_{\boldsymbol{X}} T
$$

Remark: By accounting for a few reasonable simplificative assumptions, the previous field equations have been formulated in a fully decoupled form and independently one from the other. Coupling between species diffusion, mechanics, electrostatics, and heat conduction is introduced by means of the constitutive relations, which will be derived in the following section under suitable thermodynamical considerations.

\section{Thermodynamic derivation of constitutive relations}

The constitutive equations characterizing the material properties must comply with the basic laws of thermodynamics, namely the conservation of mass, the energy balance, and the entropy principle. To this aim, we collect the energy contributions of all physical processes at first and define the body's internal energy. Next, we state the entropy principle, quantify the fluxes, and derive the corresponding form of the dissipation inequality. As described in detail in [57], the formal derivation of the electro-chemo-thermo-mechanical model relies on the thermodynamics of irreversible processes and assumes local equilibrium conditions within a small volume of material at position $\boldsymbol{X}$ [14].

The internal energy $U$ changes with contributions of heat supply $Q$, heat flux $\boldsymbol{H}$, power of deformation $\boldsymbol{P}: \dot{\boldsymbol{F}}$, mass diffusion, chemical reactions, and the presence of an electric field $\boldsymbol{E} \cdot \dot{\boldsymbol{D}}$. Thus, the rate $\dot{U}$ in the material configuration can be expressed as

$$
\dot{U}=Q-\nabla_{\boldsymbol{X}} \cdot \boldsymbol{H}_{T}+\boldsymbol{P}: \dot{\boldsymbol{F}}+\sum_{k=1}^{n} \mu_{k} \dot{c}_{k}+\sum_{j=1}^{N} \mathcal{A}_{j} \dot{\mathcal{J}}_{j}-\boldsymbol{D} \cdot \dot{\boldsymbol{E}}
$$

Both reaction affinities $\mathcal{A}_{j}$ and chemical potentials $\mu_{k}$ are referred to the material volume. The mass balance comes into play through the definition of the concentration fields (10), whereas gravity forces acting on the diffusive fluxes are neglected. Moreover, the electric term is included with a negative sign because it follows from the original term $\boldsymbol{E} \cdot \dot{\boldsymbol{D}}$ by a Legendre transform.

The local rate of the entropy per unit material volume is $\dot{S}=-\nabla_{\boldsymbol{X}} \cdot \boldsymbol{H}_{S}+\Pi_{S}$, with entropy flux $\boldsymbol{H}_{S}$ and nonnegative entropy production $\Pi_{S}$. Here we follow the classical Duhem's assumption and identify the entropy flux with the heat flux per unit temperature $\boldsymbol{H}_{T} / T$; heat supply contributes to entropy production. Consequently, we obtain for the entropy production,

$$
T \Pi_{S}=T \dot{S}+\nabla_{\boldsymbol{X}} \cdot \boldsymbol{H}_{T}-\frac{1}{T} \boldsymbol{H}_{T} \cdot \nabla_{\boldsymbol{X}} T \geq 0,
$$

where the last term expresses the dissipation by heat conduction, and the condition $-\frac{1}{T} \boldsymbol{H}_{T} \cdot \nabla_{\boldsymbol{X}} T \geq 0$ determines the direction of heat flow. Further internal dissipation, $D^{\text {int }} \geq 0$, may arise as a consequence of material damage, viscous flow, and Lorentz forces, for example. We are aware that in the case of mixtures, the Duhem's assumption on the entropy flux is too restrictive. Nonetheless, for common continuum mechanical systems, more general formulations for $\boldsymbol{H}_{S}$ result in the same thermodynamic restrictions for the constitutive equations, cf. [49].

To express the dissipation inequality (19) in terms of process energy contributions, we make use of an alternative thermodynamic potential, namely the Helmholtz free energy density $\Psi=U-T S$, obtained from (18) through a Legendre transform. Inserting its rate and (18) into (19) gives the dissipation inequality

$$
T \Pi_{S}=\boldsymbol{P}: \dot{\boldsymbol{F}}-\dot{\Psi}-S \dot{T}+\sum_{k=1}^{n} \mu_{k} \dot{c}_{k}+\sum_{j=1}^{N} \mathcal{A}_{j} \dot{\mathcal{J}}_{j}-\boldsymbol{D} \cdot \dot{\boldsymbol{E}}-\frac{1}{T} \boldsymbol{H}_{T} \cdot \nabla_{\boldsymbol{X}} T .
$$


Note that the mechanical power includes the elastic power of deformation, $\boldsymbol{P}: \dot{\boldsymbol{F}}$, and inelastic contributions that may result from internal dissipative processes, e. g., plasticity, and viscosity. The thermodynamic state of the material point is then entirely defined by $\boldsymbol{F}, T, c_{k}, \mathcal{J}_{j}, \boldsymbol{E}$, and internal variables $\boldsymbol{Z}$, which account for such dissipative phenomena.

The Helmholtz free energy density is thus a function of the form $\Psi=\Psi\left(\boldsymbol{F}, T, c_{k}, \nabla c_{k}, \mathcal{J}_{j}, \boldsymbol{E}, \boldsymbol{Z}\right)$. Taking the differential gives

$$
\begin{aligned}
\dot{\Psi}= & \frac{\partial \Psi}{\partial \boldsymbol{F}}: \dot{\boldsymbol{F}}+\frac{\partial \Psi}{\partial T} \dot{T}+\sum_{k=1}^{n}\left(\frac{\partial \Psi}{\partial c_{k}}-\nabla \cdot \frac{\partial \Psi}{\partial \nabla c_{k}}\right) \dot{c}_{k}+\sum_{k=1}^{n} \nabla \cdot\left(\frac{\partial \Psi}{\partial \nabla c_{k}} \dot{c}_{k}\right) \\
& +\sum_{j=1}^{N} \mathcal{A}_{j} \mathcal{J}_{j}+\frac{\partial \Psi}{\partial \boldsymbol{E}} \cdot \dot{\boldsymbol{E}}-\boldsymbol{Y}: \dot{\boldsymbol{Z}}
\end{aligned}
$$

where we introduced the thermodynamic forces conjugate to $\boldsymbol{Z}$,

$$
\boldsymbol{Y}=-\frac{\partial \Psi}{\partial \boldsymbol{Z}} \text {. }
$$

The chemical affinities $\mathcal{A}_{j}$ can also be derived from dissipation potentials subjected to the stoichiometric constraints, i.e., they are work conjugate to the reaction-path rates $\mathcal{J}_{j}$, cf. [18].

Additionally, we split the mechanical stress into an equilibrium part $\boldsymbol{P}_{e q}$, depending on the variables of state only, and a rate-dependent dissipative contribution $\boldsymbol{P}_{v}$, also depending on the rate of deformation $\dot{\boldsymbol{F}}$,

$$
\boldsymbol{P}=\boldsymbol{P}_{e q}\left(\boldsymbol{F}, T, c_{k}, \boldsymbol{E}, \boldsymbol{Z}\right)+\boldsymbol{P}_{v}\left(\boldsymbol{F}, T, c_{k}, \boldsymbol{E}, \boldsymbol{Z} ; \dot{\boldsymbol{F}}\right) .
$$

Inserting these relations into the dissipation inequality (20) gives

$$
\begin{aligned}
T \Pi_{S}= & \left(\boldsymbol{P}_{e q}-\frac{\partial \Psi}{\partial \boldsymbol{F}}\right): \dot{\boldsymbol{F}}+\boldsymbol{P}_{v}: \dot{\boldsymbol{F}}-\left(S+\frac{\partial \Psi}{\partial T}\right) \dot{T}+\sum_{k=1}^{n}\left(\mu_{k}-\frac{\delta \Psi}{\delta c_{k}}\right) \dot{c}_{k} \\
& -\left(\boldsymbol{D}+\frac{\partial \Psi}{\partial \boldsymbol{E}}\right) \cdot \dot{\boldsymbol{E}}-\frac{1}{T} \boldsymbol{H}_{T} \cdot \nabla_{\boldsymbol{X}} T+\boldsymbol{Y}: \dot{\boldsymbol{Z}} \geq 0 .
\end{aligned}
$$

where we denote $\delta_{c} \bullet=\partial_{c} \bullet-\nabla \cdot\left(\partial_{\nabla c} \bullet\right)$. Since inequality (24) must hold for any admissible process, the terms in brackets must vanish, leading to the equations of state (or constitutive relations) for the equilibrium stress, entropy, concentration, and electric induction:

$$
\boldsymbol{P}_{e q}=\frac{\partial \Psi}{\partial \boldsymbol{F}}, \quad S=-\frac{\partial \Psi}{\partial T}, \quad \mu_{k}=\frac{\delta \Psi}{\delta c_{k}}, \quad \boldsymbol{D}=-\frac{\partial \Psi}{\partial \boldsymbol{E}}, \quad 0=\sum_{k=1}^{n} \nabla \cdot\left(\frac{\partial \Psi}{\partial \nabla c_{k}} \dot{c}_{k}\right) .
$$

The last expression follows from mass conservation, $\sum_{k=1}^{n} \dot{c}_{k}=0$, and a restatement of $\Psi$. The surviving terms define the dissipation as

$$
T \dot{\Pi}_{S}=\boldsymbol{P}_{v}: \dot{\boldsymbol{F}}-\frac{1}{T} \boldsymbol{H}_{T} \cdot \nabla_{\boldsymbol{X}} T+\boldsymbol{Y}: \dot{\boldsymbol{Z}} \geq 0,
$$

which constrains the constitutive law for the heat flux $\boldsymbol{H}_{T}$ and the set of internal variables $\boldsymbol{Z}$. An admissible choice is Duhamel's law of heat conduction (17), which defines the heat to flow for a negative temperature gradient, Eq. (17). The general framework is completed with the definition of the viscous dissipation and the kinetic relations for $\boldsymbol{Z}$.

\section{Constitutive model for Lithium electrodes}

Here we will present the constitutive model for the electrochemical-thermomechanical processes of anode charging in lithium batteries. In particular, we reduce the process to a binary diffusion system without additional chemical reactions and refer for the latter to [56]. The normalized concentration of $\mathrm{Li}^{+}$ions is denoted as $c=c_{1}$, whereas the complementary concentration of the anode's material is $c_{2}=1-c_{1}$. 


\subsection{Kinematics}

We start by specifying the kinematics of deformation and introduce the multiplicative decomposition of the deformation gradient (1) into elastic and (several) inelastic components,

$$
\boldsymbol{F}(\boldsymbol{X}, t)=\boldsymbol{F}_{e} \boldsymbol{F}_{i} .
$$

The multiplicative decomposition (27) is a convenient mathematical representation of configurational changes of a system undergoing multi-physics processes in large deformations, [16]. It was introduced in [24,43,48] for thermoelasticity, viscoelasticity, and phenomenological elastoplasticity, respectively.

At the electrode, the inelastic deformation results from geometrical changes due to the $\mathrm{Li}^{+}$intercalation and swelling $\boldsymbol{F}_{\mathrm{S}}$, viscous distortion $\boldsymbol{F}_{v}$, thermal expansion $\boldsymbol{F}_{\text {th }}$, and the active electric deformation $\boldsymbol{F}_{\text {act }}$. We remark that the inelastic parts of the deformation take place locally and result in a non-compatible intermediate configuration. The elastic part of the deformation gradient $\boldsymbol{F}_{e}$ is related to the passive response of the material and relaxes the continuum from the intermediate to the current deformed configuration where equilibrium and compatibility conditions are fully satisfied, Fig. 1.

The swelling as a consequence of the $\mathrm{Li}^{+}$intercalation is unknown but assumed to be a purely volumetric deformation. Further inelastic mechanical deformations are identified with the isochoric viscous deformation components $\boldsymbol{F}_{v}$. Local deformations induced by the electric field are very small, cf. Sect. 5.3, which allows us to set $\boldsymbol{F}_{\text {act }}$ to unity. Thermal expansions can be stated in the form $\boldsymbol{F}_{\text {th }}=\alpha_{\mathrm{th}} \mathbb{1}+\sum_{i} a_{i} \boldsymbol{N}_{i} \otimes \boldsymbol{N}_{i}$ where $\alpha_{\text {th }}, a_{i}$ are temperature-dependent functions, cf. [48]. Because of the amorphous anode material, we assume an isotropic expansion with $a_{i}=0$ and an expansion coefficient which depends on $T$ and $c, \alpha_{\mathrm{th}}(T, c) \geq 0$. With these assumptions at hand, the multiplicative decomposition of the deformation gradient (27) can be restated as

$$
\boldsymbol{F}=J_{i}^{1 / 3} \boldsymbol{F}_{e} \boldsymbol{F}_{v}
$$

with volumetric component $\operatorname{det} \boldsymbol{F}=J_{i} \operatorname{det}\left(\boldsymbol{F}_{e} \boldsymbol{F}_{\text {th }}\right), J_{i}=\operatorname{det} \boldsymbol{F}_{i}$. The relation between the inelastic swelling $J_{i}$ and the concentration and phase segregation of intercalated lithium ions, i.e., the function $J_{i}(c)$ is unknown and must be determined. A straightforward ansatz is to assume a direct proportionality between volumetric expansion and $c,[22,30]$, as commonly done in small strain elasticity models, [21,28,63]. The finite deformation model proposed by Anand [2] suggests that only known relation is $\partial J_{i} / \partial c \geq 0$. Using a virtual-power approach, this conditions leads directly to $\dot{J}_{i}=\Omega \dot{c}$, where the constant $\Omega$ is related to the atomic volume of $\mathrm{Li}^{+}$. Xu and coworkers extended this model in order to perform numerical calculations $[66,67]$ and concluded that the inelastic swelling $J_{i}$ must be compensated by an elastic swelling $J_{e}$ to obtain a concentration-independent overall deformation as reported in [2]. This condition leads to a dependent elastic deformation $J_{e}\left(J_{i}\right)$ and

$$
J=J_{e}(c) J_{i}(c) \text { with } J_{i}=1+\Omega c .
$$

Here we propose a different model and assume $J_{i}(c)$ to be an unknown function. Then, a Taylor expansion at $c_{0}$ gives

$$
J_{i}(c)=1+\Omega\left(c-c_{0}\right)+\mathcal{O}\left(\left(c-c_{0}\right)^{2}\right)
$$

where $c_{0}$ is a reference value. The elastic volumetric deformation will relax the continuum from the intermediate configuration to the fully compatible current configuration and thus we state:

$$
J(c)=J_{e} J_{\text {th }} J_{i}(c) \text { with } J_{i}=1+\Omega\left(c-c_{0}\right) .
$$

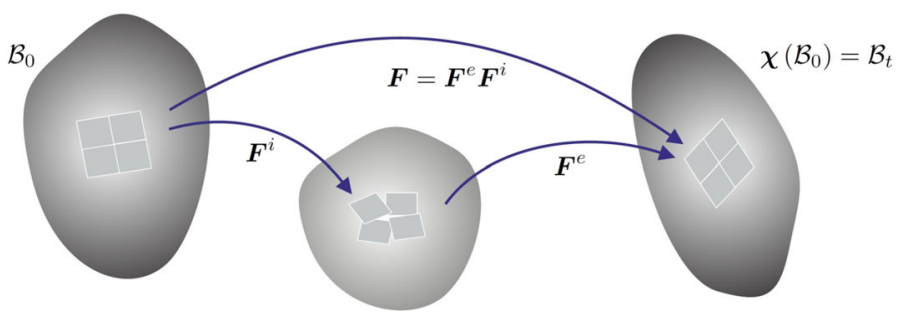

Fig. 1 Reference, current, and non-compatible intermediate configuration of the body 


\subsection{Helmholtz free energy density}

Next, we collect all the contributions to the free energy involved in the process. For ease of readability and implementation, we chose to formulate the free energy density as a function of independent variables $\boldsymbol{F}$ and $J$, cf. $[11,20]$. Moreover, we define the isochoric part of the elastic deformation gradient as $\overline{\boldsymbol{F}}_{e}=J_{e}^{-1 / 3} \boldsymbol{F}_{e}$.

\subsubsection{Elastic energy}

The sensitivity of a battery to mechanical deformation depends strongly on the material of the electrode. For example, the specific capacity of silicon is one order of magnitude larger than the one of graphite. The excellent capacity of a silicon anode is associated with a $>300 \%$ volumetric expansion during charging [9], relative to the $6-10 \%$ volume expansion observed for in graphite anode [1].

We introduce a hyperelastic material model characterized by an elastic free energy $\Psi^{e}$ independent of any dissipative internal process. Commonly, $\Psi^{e}$ is decomposed additively in volumetric and isochoric parts; thus, we formulate a neo-Hookean energy density, extended to the compressible range, of the form

$$
\begin{aligned}
\Psi^{e}\left(\boldsymbol{F}_{e}, J_{e}, T, c\right) & =\Psi^{\mathrm{iso}}\left(\boldsymbol{F}_{e}, J_{e}, T, c\right)+\Psi^{\mathrm{vol}}\left(J_{e}, c, T\right) \\
& =\frac{1}{2} G(T, c)\left(\operatorname{tr} \overline{\boldsymbol{F}}_{e}^{T} \overline{\boldsymbol{F}}_{e}-3\right)+\frac{1}{4} K(T, c)\left(J_{e}^{2}-2 \ln J_{e}-1\right)
\end{aligned}
$$

where $K$ is bulk modulus and $G$ a shear modulus. Both moduli may depend on $\mathrm{Li}^{+}$concentration and temperature. An alternative form of the hyperelastic material model, proven to be convenient in numerical applications [44-46], is the following

$$
\begin{aligned}
\Psi^{e}= & \frac{1}{2} G(T, c)\left(\operatorname{tr} \boldsymbol{F}_{e}^{T} \boldsymbol{F}_{e}-3\right)-\frac{1}{6} G(T, c)\left(J_{e}^{2}+4 \ln J_{e}-1\right) \\
& +\frac{1}{4} K(T, c)\left(J_{e}^{2}-2 \ln J_{e}-1\right) .
\end{aligned}
$$

The main advantage of the model (33) is its simplicity: It saves the decomposition of the deformation gradient and has a direct analogy to linear elasticity.

Starting from the elastic parameters of the two species, the equivalent elastic parameters to be used in (32) and (33), we apply a Vergard's mixture rule

$$
K(T, c)=K_{0}(T)(1-c)+K_{1}(T) c, \quad G(T, c)=G_{0}(T)(1-c)+G_{1}(T) c .
$$

\subsubsection{Thermal energy}

As is any chemical system, the battery state depends on the temperature. The temperature rise during charging is a slow process. Because we are expecting a small effect of the anode's design, here we consider the temperature as driven by Joule heating but not affected by the deformation. Thus, the thermo-mechanical coupling can be modeled with the classical structural entropy potential

$$
\begin{aligned}
\Psi^{\mathrm{eth}}\left(J_{e}, T, c\right) & =-\alpha_{\mathrm{th}}(c)\left(T-T_{0}\right) \frac{\partial \Psi^{\mathrm{vol}}(\boldsymbol{X}, t)}{\partial J_{e}} \\
& =-\frac{1}{2} \alpha_{\mathrm{th}}(c)\left(T-T_{0}\right) K(T, c)\left(J_{e}-J_{e}^{-1}\right),
\end{aligned}
$$

whereas the potential for the thermal entropy is

$$
\Psi^{\mathrm{temp}}(T)=C_{T}\left(T-T_{0}-T \ln \left(\frac{T}{T_{0}}\right)\right)
$$




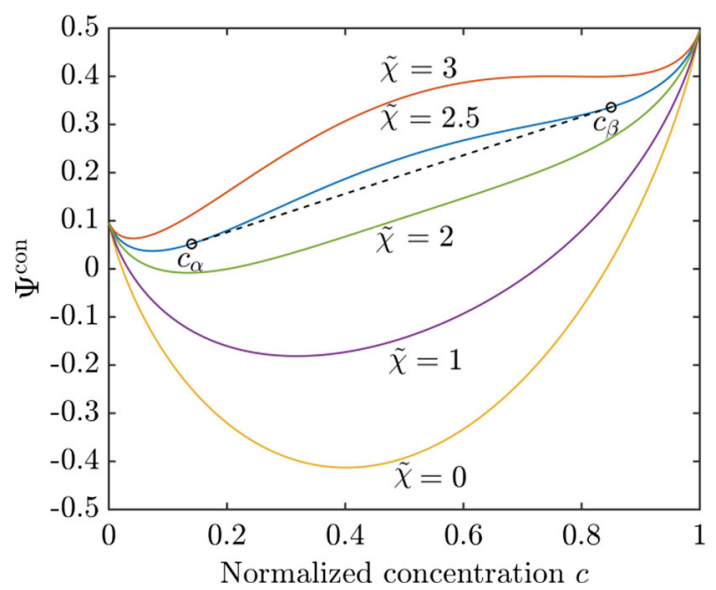

Fig. 2 Configurational energy density (38) of a binary mixture for different Flory-Huggins interaction parameters $\tilde{\chi}$. In the plot $\Psi^{\text {con }}$ is normalized with $\theta(T), g_{1}^{0}=0.1$ and $g_{2}^{0}=0.5$; for $\tilde{\chi}=2.5$ the common tangent indicates the equilibrium concentrations $c_{\alpha}$ and $c_{\beta}$

\subsubsection{Chemical energy}

For the mixing energy of the diffusion system, we assume a constant pressure which allows us to formulate the free energy in equivalence to the Gibbs free energy. The configurational free energy density $\Psi^{\text {con }}$ for a binary mixture with two equilibrium phases at the concentration $c_{\alpha}\left(\alpha\right.$-phase) and $c_{\beta}$ ( $\beta$-phase) is modeled with the common Margules form

$$
\begin{aligned}
\Psi^{\mathrm{con}}(T, c)= & g_{1}(1-c)+g_{2} c+\theta(T)(c \ln (c)+(1-c) \ln (1-c)) \\
& +\theta(T) \tilde{\chi} c(1-c) .
\end{aligned}
$$

Here $g_{1}$ and $g_{2}$ are Gibbs free energy densities of both components. The third term accounts for energy contributions from the entropy of mixing. According to the classical Lewis-Randall ideal-solution model, the entropy contribution is typically weighted by a temperature-dependent material function $\theta(T)$, e.g., $\theta(T)=$ $R T y_{\text {ref }}$, where $y_{\text {ref }}$ is reference molar concentration.

For non-ideal mixtures, the energy must include an excess energy contribution that describes the decomposition of a non-ideal mixture into distinct phases. Here, the excess energy is chosen to be of Porter type [see [33]], with the Flory-Huggins interaction parameter $\tilde{\chi}$. For $\tilde{\chi}<2$, the mixing energy (38) is convex and corresponds to the energy of a homogeneous solution. Phase separation will be observed only for $\tilde{\chi}>2$, when the configurational energy turns into a double-well function, i.e., it has two relative minima and a concave (spinodal) region in between. The concentrations $c_{\alpha}$ and $c_{\beta}$ are the binodal points where $\partial_{c} \Psi^{\text {con }}\left(c_{\alpha}\right)=\partial_{c} \Psi^{\text {con }}\left(c_{\beta}\right)$. The situation is illustrated for different values of $\tilde{\chi}$ in Fig. 2. density

Furthermore, the coexistence of phases causes additional energy contributions. The interfacial free energy

$$
\Psi^{\mathrm{int}}(\nabla c, T)=\frac{\kappa(T)}{2}\|\nabla c\|^{2},
$$

accounts for the nonlocal effect of surface tension with interface tension parameter $\kappa>0$.

\subsubsection{Electric energy contribution}

For the effect of the electric induction on the material, we introduce the active part of the Helmholtz free energy. With $\boldsymbol{E}=\boldsymbol{F}^{\top} \boldsymbol{e}$ and assuming a standard dielectric with permittivity $\varepsilon_{\mathrm{e}}$, it reads

$$
\Psi^{\mathrm{act}}(\boldsymbol{F}, \boldsymbol{E}, c)=-\frac{1}{2} \varepsilon_{\mathrm{e}}(c) J \boldsymbol{E} \boldsymbol{F}^{-1} \cdot \boldsymbol{F}^{-\top} \boldsymbol{E} .
$$


The thermo-electric coupling reduces to resistive heat generation; other thermoelectric effects like described in [40] are not expected here. The electric power determines Joule heating. For a given electric field $\boldsymbol{E}$, it has the potential

$$
\Psi^{\mathrm{jou}}(T, \boldsymbol{E}, c)=-\frac{\alpha T}{R_{\mathrm{m}} T_{\mathrm{ref}}}\left(J \boldsymbol{E} \boldsymbol{F}^{-1} \cdot \boldsymbol{F}^{-\top} \boldsymbol{E}\right)
$$

where $R_{\mathrm{m}}$ is the material's resistivity and $\alpha \leq 1$ a conversion factor.

\subsubsection{Inelasticity and viscosity}

In the charging process of the electrode, some inelastic mechanical deformations may occur, cf. [10]. Within a variational framework, the existence of a free energy potential is assumed such that the inelastic driving forces are derived as work conjugate to the inelastic part of the deformation, e.g.,

$$
\boldsymbol{Y}=-\frac{\partial \Psi}{\partial \boldsymbol{F}_{i}}
$$

and further forces are conjugate to the internal variables, Eq. (22). Additionally, a rate-dependent potential $\Phi$ may be introduced to characterize the rate-dependent dissipative processes. The rate of the inelastic deformations is subjected to the kinematic restrictions imposed by a flow rule, and for the evolution of the internal variables, suitable kinetic equations must be supplied. This technique leads to variational constitutive relations, as described in $[6,34,59]$ for plasticity and viscoelasticity.

From the typical cyclic loading of an electrode, we can interpret the inelastic mechanical deformation as viscous, i.e., the material state depends on the rate of deformation $\dot{\boldsymbol{F}}$. The equilibrium stress state is defined as $\boldsymbol{P}_{\mathrm{eq}}=\boldsymbol{P}(\dot{\boldsymbol{F}}=0)$. The viscous stress is derived from rate-dependent free energy contribution $\Psi^{\mathrm{vis}}$ such that

$$
\boldsymbol{P}_{v}=\frac{\partial \Psi^{\mathrm{vis}}(\dot{\boldsymbol{F}}, \boldsymbol{F}, \ldots)}{\partial \dot{\boldsymbol{F}}} .
$$

Here we choose viscosity to be volume preserving Newtonian. Specifically, we refer to the common generalized Maxwell model composed of $N$ spring-dashpot elements in parallel, each with shear modulus $G_{j}$, viscosity $\eta_{j}$, and relaxation time $\tau_{j}=\eta_{j} / G_{j}$. The contribution to the body's free energy density is then

$$
\Psi^{\mathrm{vis}}(\dot{\boldsymbol{F}}, \boldsymbol{F}, T, c)=\sum_{j=1}^{N} \Psi_{j}^{\mathrm{vis}}(\dot{\boldsymbol{F}}, \overline{\boldsymbol{F}}, T, c),
$$

where for each of the Maxwell elements, the rate-depended energy density is

$$
\Psi_{j}^{\mathrm{vis}}\left(\dot{\boldsymbol{F}}_{v}, \overline{\boldsymbol{F}}, T, c\right)=\frac{\eta_{j}}{2}(T, c)\left(\dot{\boldsymbol{F}}_{j}^{v \top}: \overline{\boldsymbol{F}}_{j}^{v}+\overline{\boldsymbol{F}}_{j}^{v \top}: \dot{\boldsymbol{F}}_{j}^{v}\right)
$$

In (45), $\boldsymbol{F}_{j}^{v}$ denotes the viscous part of the deformation gradient of the $j$-th Maxwell element which follows the evolution equation

$$
\boldsymbol{F}_{j}^{v}+\tau_{j} \dot{\boldsymbol{F}}_{j}^{v}=\overline{\boldsymbol{F}}(t) \quad j=1, \ldots, N .
$$

The total $\boldsymbol{F}^{v}$ is the weighted sum of all elements in time and leads to the solution

$$
\boldsymbol{F}^{v}(t)=\sum_{j=1}^{N} \int_{0}^{t} \frac{G_{j}}{G} \exp \left(-\frac{t-s}{\tau_{j}}\right) \dot{\boldsymbol{F}}^{v}(s) \mathrm{d} s .
$$

We remark that our model corresponds to the classical hereditary integral theory of linear viscoelasticity [52] and can conveniently be solved with the help of a recursive scheme. For simplicity, we assume the moduli $G_{j}$ and $\eta_{j}$ to depend on $c$ and $T$ in the same way, i.e., $\tau_{j}$ is independent of concentration and temperature. 


\subsubsection{Summary}

The total Helmholtz free energy density of the anode material has the following expression:

$$
\begin{aligned}
\Psi\left(\boldsymbol{F}_{e}, J_{e}, c, \nabla c, T, \boldsymbol{E}\right)= & \Psi^{\mathrm{iso}}\left(\boldsymbol{F}_{e}, J_{e}, c, T\right)+\Psi^{\mathrm{vol}}\left(J_{e}, c, T\right)+\Psi^{\mathrm{con}}(T, c) \\
& +\Psi^{\mathrm{temp}}(T)+\Psi^{\mathrm{jou}}(T, \boldsymbol{E}, c)+\Psi^{\mathrm{eth}}\left(J_{e}, c, T\right) \\
& +\Psi^{\mathrm{int}}(\nabla c, T)+\Psi^{\mathrm{act}}(\boldsymbol{F}, \boldsymbol{E}, c)+\Psi^{\mathrm{vis}}(\dot{\boldsymbol{F}}, \boldsymbol{F}, T, c) .
\end{aligned}
$$

\subsection{State equations}

\subsubsection{Mechanical stresses}

The first Piola-Kirchhoff stress tensor follows from the constitutive relation (25) $)_{1}$ as the work conjugate to the deformations gradient. In equilibrium, it is

$$
\boldsymbol{P}=\frac{\partial \Psi}{\partial \boldsymbol{F}}=\boldsymbol{P}_{e} \boldsymbol{F}_{i}^{-\top}
$$

and additional rate-dependent stress contributions may apply, Eq. (23). With decomposition (27), the isochoric expression $\overline{\boldsymbol{F}}_{e}$ and involving the corresponding derivatives using the chain rule, we obtain for $\boldsymbol{P}_{e}$

$$
\begin{aligned}
\boldsymbol{P}_{e} & =\frac{\partial \Psi}{\partial \boldsymbol{F}_{e}}+\frac{\partial \Psi}{\partial J_{e}} \frac{\partial J_{e}}{\partial \boldsymbol{F}_{e}} \\
& =\frac{\partial \Psi^{\text {iso }}}{\partial \overline{\boldsymbol{F}}_{e}}\left(J_{e}^{-1 / 3}\right)+\left(\frac{\partial \Psi^{\text {iso }}}{\partial \overline{\boldsymbol{F}}_{e}}:\left(-\frac{1}{3} J_{e}^{-4 / 3}\right) \boldsymbol{F}_{e}+\frac{\partial \Psi^{\mathrm{vol}}}{\partial J_{e}}\right) J_{e} \boldsymbol{F}_{e}^{-\top}
\end{aligned}
$$

The resulting equilibrium stress of Eq. (49) is the pulled-back elastic stress. Defining a conjugate stress $\overline{\boldsymbol{P}}=\partial \Psi^{\text {iso }} / \partial \overline{\boldsymbol{F}}_{e}$ and a pressure term $p=\partial \Psi^{\mathrm{vol}} / \partial J_{e}$ we re-state it as

$$
\boldsymbol{P}=\left(J_{e}^{-1 / 3} \overline{\boldsymbol{P}}+\left(-\frac{1}{3} J_{e}^{-4 / 3} \overline{\boldsymbol{P}}: \boldsymbol{F}_{e}+p\right) J_{e} \boldsymbol{F}_{e}^{-\top}\right) \boldsymbol{F}_{i}^{-\top}
$$

which illustrates that any ansatz for the hyperelastic energy density just enters the expressions for $\overline{\boldsymbol{P}}$ and/or $p$.

For the specific free energy model (32), we evaluate the first Piola-Kirchhoff stress tensor

$$
\begin{aligned}
\boldsymbol{P}= & {\left[\frac{1}{2} K\left(\left(J^{2}-1\right)-\alpha_{\mathrm{th}}\left(T-T_{0}\right)\left(J_{e}-J_{e}^{-1}\right)\right) \boldsymbol{F}_{e}^{-\top}\right.} \\
& \left.+G J_{e}^{-2 / 3}\left(-\frac{1}{3} \operatorname{tr}\left(\boldsymbol{C}_{e}\right) \boldsymbol{F}_{e}^{-\top}+\boldsymbol{F}_{e}\right)\right] \boldsymbol{F}_{i}^{-\top}+\sum_{j=1}^{N} \eta_{j} \dot{\boldsymbol{F}} \\
& -\left(\frac{\alpha T}{R_{\mathrm{m}} T_{\mathrm{ref}}}+\varepsilon_{e}\right) J\left[\boldsymbol{E} \boldsymbol{F}^{-1} \otimes \boldsymbol{F}^{-\top} \boldsymbol{E}-\frac{1}{2}\left(\boldsymbol{E} \boldsymbol{F}^{-1} \cdot \boldsymbol{F}^{-\top} \boldsymbol{E}\right) \mathbb{1}\right] \boldsymbol{F}^{-\top} .
\end{aligned}
$$

For model (33), the first term of (52) changes, and we obtain the mechanical stress

$$
\begin{aligned}
\boldsymbol{P}= & {\left[G\left(\boldsymbol{F}_{e}-\boldsymbol{F}_{e}^{-\top}\right)+\frac{1}{2} K\left(\left(J^{2}-1\right)-\alpha_{\mathrm{th}}\left(T-T_{0}\right)\left(J_{e}-J_{e}^{-1}\right)\right) \boldsymbol{F}_{e}^{-\top}\right] \boldsymbol{F}_{i}^{-\top} } \\
& -\left(\frac{\alpha T}{R_{\mathrm{m}} T_{\mathrm{ref}}}+\varepsilon_{e}\right) J\left[\boldsymbol{E} \boldsymbol{F}^{-1} \otimes \boldsymbol{F}^{-\top} \boldsymbol{E}-\frac{1}{2}\left(\boldsymbol{E} \boldsymbol{F}^{-1} \cdot \boldsymbol{F}^{-\top} \boldsymbol{E}\right) \mathbb{1}\right] \boldsymbol{F}^{-\top} \\
& +\sum_{j=1}^{N} \eta_{j} \dot{\boldsymbol{F}}
\end{aligned}
$$

Clearly, the coupling of diffusion, temperature, electric field, and elasticity manifest itself in the different stress contributions (and also in the field-dependent material moduli). The first lines of (52) and (53) include 
the thermo-mechanical stresses; next follows the viscous stress contribution $\boldsymbol{P}_{\boldsymbol{v}}$, and the last terms account for the presence of an electric field, which is usually referred to as the Maxwell stress and its heating contribution. The Cauchy stress $\sigma$ in the current configuration can be obtained by a push-forward operation, according to Eq. (15).

\subsubsection{Thermal field}

The entropy field $(25)_{2}$ is conjugate to the temperature $T$ with the result

$$
\begin{aligned}
S= & \frac{1}{2}\left(\left(\alpha_{\mathrm{th}}(T, c)+\frac{\partial \alpha_{\mathrm{th}}}{\partial T} T\right) K+\alpha_{\mathrm{th}}(T, c)\left(T-T_{0}\right) \frac{\partial K}{\partial T}\right)\left(J-J^{-1}\right) \\
& +C_{T} \ln \left(\frac{T}{T_{0}}\right)-\frac{\partial \theta}{\partial T}(c \ln (c)+(1-c) \ln (1-c)+\tilde{\chi} c(1-c))-\frac{1}{2} \frac{\partial \kappa}{\partial T}\|\nabla c\|^{2} \\
& +\left(\left(\frac{1}{2} \varepsilon_{\mathrm{e}}+\frac{\alpha T}{R_{\mathrm{m}} T_{\mathrm{ref}}}\right) \frac{\partial J}{\partial T}+\frac{\alpha}{R_{\mathrm{m}} T_{\mathrm{ref}}} J\right) \boldsymbol{E}^{-1} \cdot \mathbf{F}^{-\top} \boldsymbol{E} \\
& -\frac{1}{4} \frac{\partial K}{\partial T}\left(J_{e}^{2}-2 \ln \left(J_{e}\right)-1\right)-\frac{1}{2} \frac{\partial G}{\partial T}\left(\operatorname{tr}\left(\mathbf{F}_{e}^{\top} \mathbf{F}_{e}\right)-3\right) .
\end{aligned}
$$

The fact that $S=0$ for $T \rightarrow 0$ needs to be considered in the constants $\alpha_{\mathrm{th}}(T), G(T), \ldots$ but is of no relevance here.

\subsubsection{Chemical potential}

The chemical potential of a species follows from $(25)_{3}$. It expresses the free energy change for adding or removing one mole of a component to or from the system; thus, it is the same for any configuration. With energy (48), we obtain

$$
\begin{aligned}
\mu= & \frac{1}{2} \frac{\partial G}{\partial c}\left(\operatorname{tr}\left(\overline{\mathbf{F}}_{e}^{\top} \overline{\mathbf{F}}_{e}\right)-3\right)+\frac{1}{4} \frac{\partial K}{\partial c}\left(J_{e}^{2}-2 \ln J_{e}-1\right) \\
& -\frac{1}{2}\left(\frac{\partial \alpha_{\mathrm{th}}}{\partial c} K+\alpha_{\mathrm{th}} \frac{\partial K}{\partial c}\right)\left(T-T_{0}\right)\left(J_{e}-J_{e}^{-1}\right) \\
& +g_{1}-g_{2}+\theta(T)\left(\ln \frac{c}{1-c}+\tilde{\chi}(1-2 c)\right)-\kappa(T) \Delta c \\
& -\left(\frac{\alpha T}{R_{\mathrm{m}} T_{\mathrm{ref}}} \frac{\partial J}{\partial c}+\frac{1}{2}\left(\frac{\partial \varepsilon_{\mathrm{e}}}{\partial c} J+\varepsilon_{\mathrm{e}} \frac{\partial J}{\partial c}\right)\right) \boldsymbol{E F}^{-1} \cdot \mathbf{F}^{-\top} \boldsymbol{E}
\end{aligned}
$$

where we use (52) (or alternatively (53)) for the derivative of the elastic energy. Moreover, $\partial K / \partial c$ is the derivative of the overall elastic bulk modulus function (34) with respect to the corresponding concentration; the same holds for $G, \alpha_{\mathrm{th}}, \ldots$. These terms vanish for constant elastic parameters. Obviously, the first two lines in (55) include the coupling of mass diffusion and the thermoelastic field; the third line comes from the evolution of the chemical field and the thermo-electro-chemical coupling gives the fourth line.

\subsubsection{Electric field contribution}

The constitutive Eq. (25) 4 provides the electric induction as

$$
\boldsymbol{D}=\left(\frac{\alpha T}{R_{\mathrm{m}} T_{\mathrm{ref}}}+\varepsilon_{e}\right) J\left(\mathbf{F}^{\top} \mathbf{F}\right)^{-1} \boldsymbol{E}
$$

and corresponds to (16) for isothermal conditions. 


\section{Properties of the coupled model}

This subsection presents characteristic features of our coupled electrochemical-thermomechanical model. For clarity, univariate parameter studies are performed and, because a thermo-mechanical material behavior is well investigated, the focus is set here on the chemo-mechanical and electro-mechanical interaction. Additionally, we provide a comparison to the finite deformation model of Anand [2] and Xu [66].

\subsection{Chemical energy, phase decomposition and mechanical properties}

We consider two material states with bulk moduli $K_{0}=K(c=0)$ and $K_{1}=K(c=1)$. Depending on the electrode material, these values may be rather similar, e.g., $K_{1} \approx 0.9 K_{0}$ for a typical cathode material like $\mathrm{Li}_{x} \mathrm{Mn}_{2} \mathrm{O}_{4}$ [25], or quite different, e.g., $K_{1} \approx 0.05 K_{0}$ for the Li-Si mixture in a silicon anode [27,42]. For both situations, the influence on the equilibrium concentrations $c_{\alpha}^{\mathrm{eq}}$ and $c_{\beta}^{\mathrm{eq}}$ is studied.

The Helmholtz free energy density (48) follows here from Eqs. (32), (34) and (38) with $g_{1}=g_{2}=0$ and a material-dependent normalization by $\theta$ in the dimensionless form

$$
\Psi=c \ln (c)+(1-c) \ln (1-c)+\tilde{\chi} c(1-c)+\frac{1}{2 \theta} J_{i}\left(\frac{1}{J_{i}}-1\right)^{2}\left(K_{0}(1-c)+K_{1} c\right) .
$$

For further investigation, we set $\theta=10 \mathrm{kN} / \mathrm{mm}^{2}$ and the Flory-Huggins parameter to $\tilde{\chi}=2.5$, cf. Fig. 2 . The chemical potential results from the total derivative wrt. $c$, Eq. (25). A mechanically frozen state is considered, $\mathbf{F}=\mathbb{1}$, and following Cahn-Hilliard's theory of phase decomposition, the common tangent construction is used to determine the equilibrium concentrations. Specifically, the concentrations $c_{\alpha}$ and $c_{\beta}$ are calculated with a bisection algorithm for the coupled system of equations

$$
\begin{aligned}
& 0=\mu_{\alpha}-\mu_{\beta} \\
& 0=\Psi_{\alpha}-\Psi_{\beta}-\mu_{\alpha}\left[c_{\alpha}^{\mathrm{eq}}-c_{\beta}^{\mathrm{eq}}\right]
\end{aligned}
$$

where $\Psi_{\alpha}=\Psi\left(c=c_{\alpha}^{\mathrm{eq}}\right), \Psi_{\beta}=\Psi\left(c=c_{\beta}^{\mathrm{eq}}\right), \mu_{\alpha}=\mu\left(c=c_{\alpha}^{\mathrm{eq}}\right)$ and $\mu_{\beta}=\mu\left(c=c_{\beta}^{\mathrm{eq}}\right)$. Clearly, both $\mu$ and $\Psi$ depend on the inelastic volume swelling $J_{i}$ in a nonlinear way.

We recall that we approximate $J_{i}=1+\Omega\left(c-c_{0}\right)$ according to Eq. (30), i.e., there is no inelastic deformation when $c=c_{0}$. Here, we set $\Omega=0.8$. The Taylor series expansion at reference point $c_{0}$ determines the reference configuration and thus affects the shape of the potentials $\Psi(c)$. At first, we expand $J_{i}(c)$ at $c_{0}=0$ (as done in the models of $[2,26,66])$. The resulting double well potentials $\Psi(c)$ are displayed in the left plot of Fig. 3. We see that a higher value $\Delta K=\left(K_{0}-K_{1}\right) / \theta$ straightens and shifts the $\Psi(c)$-curve which means that a high difference of $K_{0}$ and $K_{1}$ works against phase decomposition. In other words, phase segregation can be suppressed by a large enough elastic modulus.

Also a different reference value $c_{0}$ shifts the positions of the equilibrium concentrations $c_{\alpha, \beta}^{\mathrm{eq}}$ and changes the shape of the $\Psi(c)$ curve. In the central plot of Fig. 3, we have $c_{0}=0.5$ and see already a convex energy $\Psi$ for $\Delta K=0.4$; only small values and differences in $K$ lead to a double well potential. The situation is even more obvious for the limit case $c_{0}=1$ where both curves are convex, see left plot of Fig. 3 .
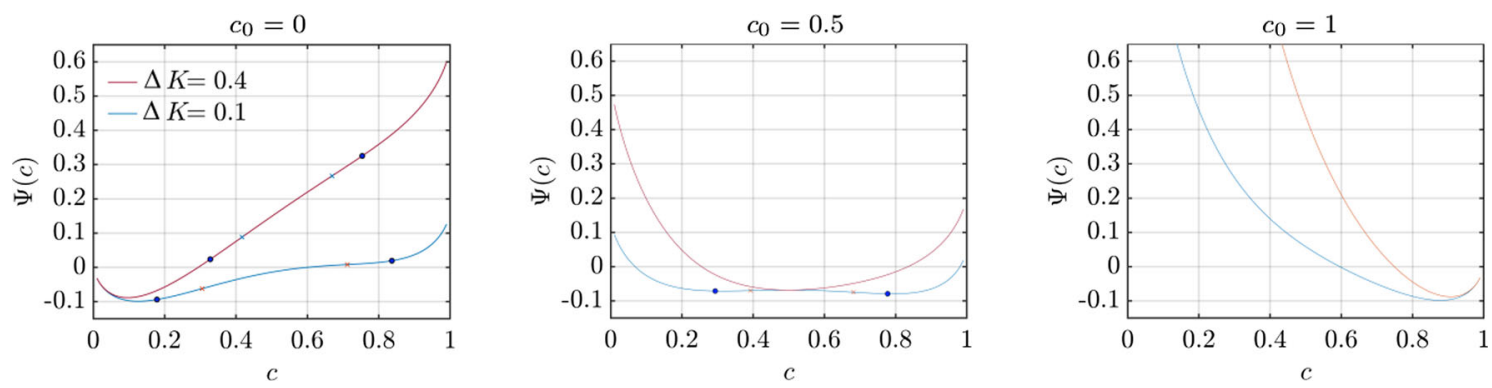

Fig. 3 Free energy potential of Eq. (57) for different elastic moduli with $\Delta K=0.4$ (blue line) and $\Delta K=0.1$ (red line). The potential is displayed for expansion point $c_{0}=0$ (left), $c_{0}=0.5$ (middle) and $c_{0}=1$ (right). If existent, equilibria are marked by blue dots and spinodal points by red crosses (color figure online) 

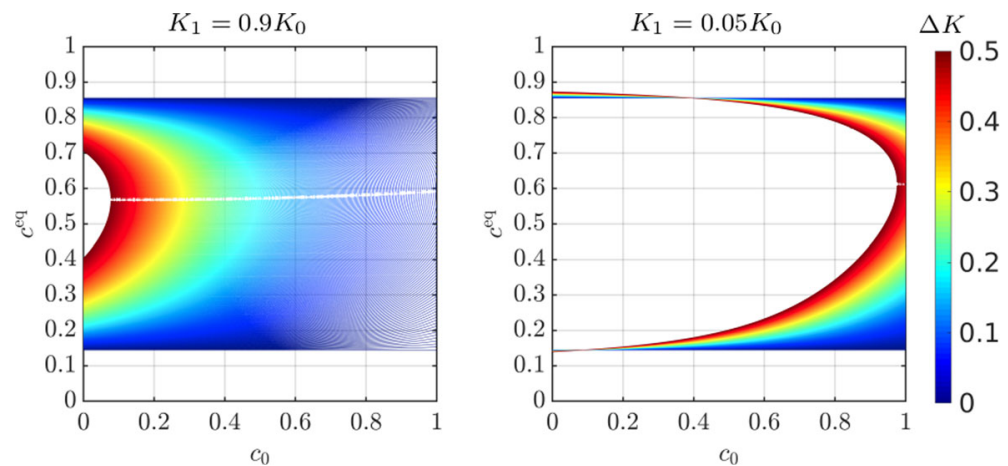

Fig. 4 Equilibrium concentrations for potential (57), which is based on concentration dependent bulk moduli with similar values of $K_{0}$ and $K_{1}$ (left) and strong softening (right). The upper branch of the plots shows $c_{\beta}$, the lower branch $c_{\alpha}$, the white stripe at $c^{\mathrm{eq}} \sim 0.6$ separates the equilibria. Color coding indicates the bulk moduli difference $\Delta K=\left(K_{0}-K_{1}\right) / \theta$ (color figure online)
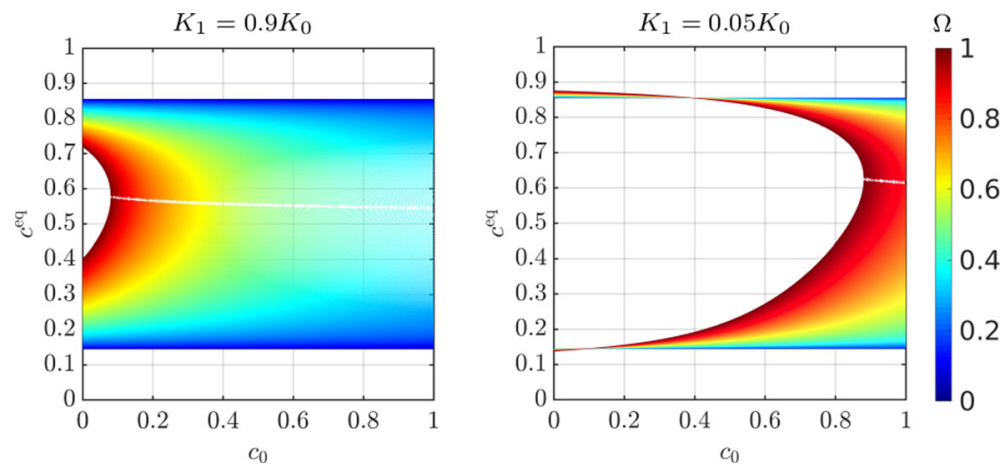

Fig. 5 Equilibrium concentrations for potential (57), which is based on concentration-dependent bulk moduli with similar values of $K_{0}$ and $K_{1}$ (left) and strong softening (right). The upper branch of the plots shows $c_{\beta}$, the lower branch $c_{\alpha}$, and the white stripe at $c^{\mathrm{eq}} \sim 0.6$ separates the equilibria. Color coding indicates the numerical value of the normalized molar volume $\Omega$ (color figure online)

Table 2 Material parameters used in the simulation

\begin{tabular}{llllllll}
\hline $\begin{array}{l}\mathrm{RT} \\
(\mathrm{kJ} / \mathrm{mol})\end{array}$ & $\begin{array}{l}y_{\max } \\
\left(\mathrm{mol} / \mathrm{m}^{3}\right)\end{array}$ & $\begin{array}{l}\Omega \\
\left(\mathrm{m}^{3} / \mathrm{mol}\right)\end{array}$ & $\begin{array}{l}K_{0}, G_{0} \\
(\mathrm{GPa})\end{array}$ & $\begin{array}{l}K_{1}, G_{1} \\
(\mathrm{GPa})\end{array}$ & $\begin{array}{l}\ell \\
(\mu \mathrm{m})\end{array}$ & $\begin{array}{l}D \\
\left(\mathrm{~m}^{2} / \mathrm{s}\right)\end{array}$ & $\begin{array}{l}\kappa \\
(\mathrm{nJm} /\end{array}$ \\
\hline 2.35 & $2.3 \times 10^{5}$ & $3.5 \times 10^{-6}$ & 10 & 9 & 1 & $7 \times 10^{-15}$ & 0.1 \\
\hline
\end{tabular}

We conclude that concentration-dependent bulk moduli and also the reference concentration for Taylor series expansion may qualitatively change the chemical energy. To illustrate the effect over the full range of $c_{0} \in[0,1]$ the equilibrium concentrations are plotted in Fig. 4 for two values of $\Delta K$. The outer blue lines correspond to $K=0$, i.e., no elastic effects on the equilibrium concentrations. There are always two phases, here with values $c_{\alpha, \beta}^{\mathrm{eq}}=0.5 \pm 0.355$. The dark red lines correspond to a high bulk modulus and indicate two equilibrium phases only for small values of $c_{0}$; for larger concentration, the elastic compression suppresses phase decomposition. The white stripe in the middle separates the equilibrium states. The situation is different for the strongly softening material in the right plot of Fig. 4. When $K_{1} \ll K_{0}$, two equilibrium phases exits also under large compression.

Similar results can be obtained for an increasing relative atomic volume $\Omega$ of the intercalating ions, see Fig. 5. Here we set $K_{0}$ and $K_{1}$ as in Table 2 and observe that a high bulk modulus, rather insensitive to concentration, suppresses phase decomposition (left), whereas strongly softening material decomposes even for 'large' intercalating ions (right).

In summary, it can be said that the equilibrium concentration strongly depends on the material parameters and also on the choice of reference value $c_{0}$. The proper choice of $c_{0}$ would be the same value employed to measure the relative atomic volume $\Omega$. For practical application, it should be set to the initial concentration. We remark that, for other choices, numerical problems may arise, because a system with reference configuration 

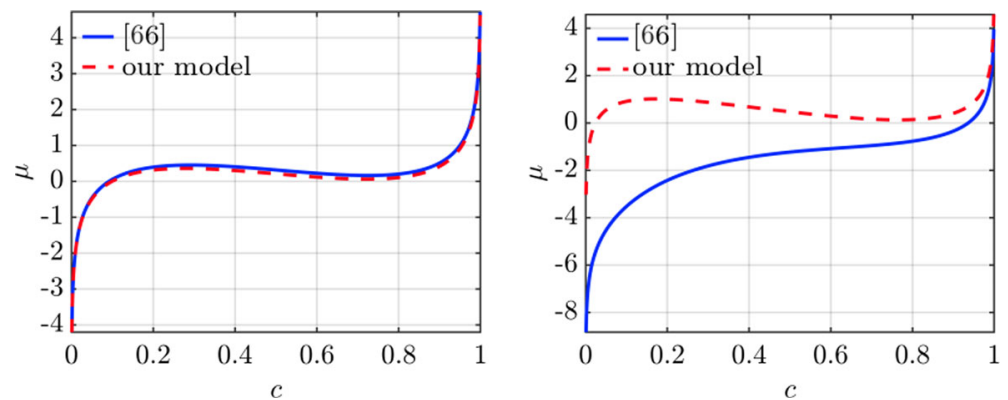

Fig. 6 Comparison of chemical potentials $\mu$ for proposed and existing model for uniaxial stretch (left) and spherical expansion (right); setting: $\lambda=1.5, K_{0} / \theta=1, K_{1} / \theta=0.9$ and $c_{0}=0$

$J_{i}=1$, initial concentration $c_{\text {ini }}$ and given relative atomic volume $\Omega$ is over-constrained and inconsistent for $c_{\text {ini }} \neq c_{0}$.

\subsection{Comparison to other chemo-mechanical models}

Anand [2] suggests a constitutive model that couples ion diffusion with large elastic and inelastic deformations of the material. This model is derived from a microforce balance theory, introduces a Mandel stress in the chemical potential, and uses phenomenological plasticity to account for inelastic effects. In the corresponding numerical implementations [26,38], several simplifications are made, e.g., the material parameters are treated as concentration independent quantities. In order to conduct comprehensive numerical simulations, $\mathrm{Xu}$ et al. [66] extended the model to include configurational changes, concentration-dependent elastic moduli, and a Lagrangian description of the constitutive equations. In the following, we compare Xu et al. [66] approach to our model.

Anand's model [2] relies on the multiplicative decomposition of $\boldsymbol{F}=\boldsymbol{F}_{e} \boldsymbol{F}_{i}$, where $\boldsymbol{F}$ accounts for elastic and inelastic mechanical deformations, but disregards temperature or electric effects. Macroscopic and microscopic force balances are formulated in the current configuration, and the principle of virtual power is used to derive evolution equations for the unknown quantities $\dot{\chi}, \dot{F}^{e}, \dot{c}$, and others. For the inelastic Jacobian, this procedure gives $\dot{J}_{i}=\Omega \dot{c}$, supporting the conclusion that $J_{i}$ has the form of Eq. (29) [67], i.e., the inelastic Jacobian is set to $J_{i}(c)=1+\Omega c$ ( $c_{0}=0$ in our model). Unlike in our model, in [67] the evolution of $J_{i}$ is assumed to be completely compensated by the elastic swelling $J_{e}$. This, in turn, follows from the underlying assumption that the right-Cauchy Green tensor $\boldsymbol{C}=\boldsymbol{F}^{T} \boldsymbol{F}$ is independent of the concentration, namely $\boldsymbol{C}=\boldsymbol{C}_{e}(c) \boldsymbol{C}_{i}(c)$.

As a consequence, the elastic free energy refers to the intermediate configuration. Expressed in terms of the neo-Hookean energy density (32), this leads to the form $\Psi^{e}=J_{i}\left(\Psi^{\mathrm{iso}}+\Psi^{\mathrm{vol}}\right)$. The derivation of the Piola-Kirchhoff stresses requires the application of the chain rule; thus, an additional term $J_{i}^{\prime} \Psi^{\mathrm{e}}$ arises from the configurational change. The remaining part of the stress is nearly the same as the first term in Eq. (52), because $J_{i} \Psi^{\mathrm{e}}$ compares with modified elastic constants in $\Psi^{\mathrm{e}}$.

Clearly, our model adopts a different kinematics. To show the differences between the models, Fig. 6 plots the chemical potential for different mechanical deformations. The curves on the left plot refer to uniaxial loading at different stretches, revealing a minor difference in the response. Our numerical experience allows to state that the results of the microforce model are very similar to the ones of our model. The observed difference in quantitative terms (i.e., magnitude of the stress) cannot be assessed because of the lack of reference data.

\subsection{Electro-mechanical coupling}

Using a standard finite element model, we want to assess the relevance of electro-mechanical coupling under realistic working conditions in a $\mathrm{Li}$-ion anode. Typical lithiation potentials have been reported to be in the range of few $\mathrm{mV}[15,32]$. Experimentally, silicon-based half cells are subjected to open-circuit voltages of $10 \mathrm{mV}$ and lithiate for $24 \mathrm{~h} \mathrm{[60].} \mathrm{Exceeding} \mathrm{a} \mathrm{threshold} \mathrm{potential} \mathrm{leads} \mathrm{to} \mathrm{a} \mathrm{significant} \mathrm{reduction} \mathrm{in} \mathrm{cycling}$ efficiency and to the failure of the solid-electrolyte interface [61]. 

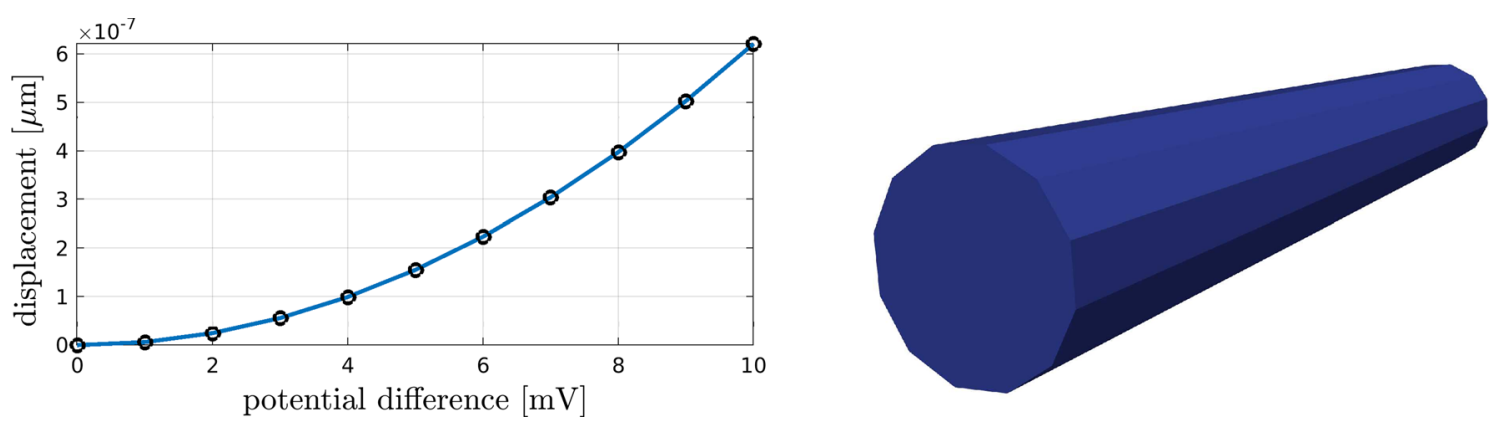

Fig. 7 Displacement of the cylinder surface under an electric field

We consider a Li-ion anode subject to a electric potential growing with $1 \mathrm{mV}$ steps up to a maximum value of $10 \mathrm{mV}$. The specimen is cylindrical with a radius of $0.5 \mu \mathrm{m}$ and a length of $7 \mu \mathrm{m}$, clamped at the two extremities, Fig. 7. The linear momentum balance (5) and the electrical induction (7) are coupled in a weak form and solved with a staggered approach [35,54]. Both bulk modulus and shear modulus are set to $10 \mathrm{GPa}$; the relative permittivity is $\varepsilon_{r}=0.15$.

The growing potential causes a growing active (inelastic) deformation, which is constrained in the longitudinal direction and free in the radial direction. The cylinder expands the radius increasing quadratically with increasing electric potential, see Fig. 7. Nevertheless, the radial displacement remains very small, with a maximum strain of $\approx 10^{-6}$. We conclude that the local deformations induced by the electric field are negligible, which justifies the choice to set $\boldsymbol{F}_{\text {act }}$ to unity, Eq. 28 .

\section{Numerical examples}

The multi-field problem described in the previous section has been solved by means of a finite element approach. The method requires to formulate the governing equations in weak form and to introduce a spatial discretization by means of approximation functions. In the following, we state the weak form of the balance equations for the coupled problem, and present a selection of illustrative numerical examples concerning elastic and inelastic phenomena in a cylindrical structure. The material parameters used in the simulations are summarized in Table 2.

\subsection{Finite element approximation}

The weak form of a differential equation requires the multiplication of the equation by a suitable test function (or variation) having the same mathematical features of the unknown field. The resulting scalar function is integrated over the physical domain, making use of the Green's formula and including natural boundary conditions.

The weak form of the mechanical problem is obtained from the material form of the linear momentum, Eq. (5), using the variation $\delta \chi$

$$
\int_{\mathcal{B}_{0}} \boldsymbol{P}: \nabla(\delta \chi) \mathrm{d} V-\int_{\mathcal{B}_{0}} \delta \chi \cdot \boldsymbol{B} \mathrm{d} V-\int_{\partial \mathcal{B}_{0}^{T}} \delta \chi \overline{\boldsymbol{T}} \mathrm{d} A=0
$$

The deformation $\chi(x, t)$ is dependent on concentration, temperature, and electric fields through the stress, Eq. (52).

The weak form of the heat equation is derived through the variation $\delta T$ as

$$
\int_{\mathcal{B}_{0}} \delta T T \dot{S} \mathrm{~d} V-\int_{\mathcal{B}_{0}} \boldsymbol{Q} \cdot \nabla(\delta T) \mathrm{d} V-\int_{\partial \mathcal{B}_{0}^{H}} \delta T \boldsymbol{H}_{T} \cdot \boldsymbol{N} \mathrm{d} A=0
$$

where entropy (54) and heat flux boundary $\partial \mathcal{B}_{0}^{H}$ are accounted for. 


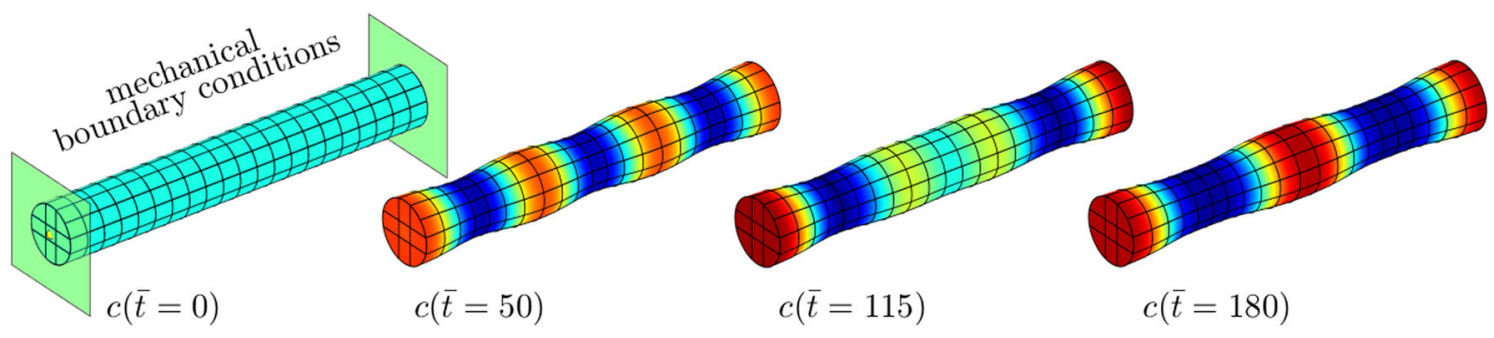

Fig. 8 Finite element model of the clamped elastic rod with an initially uniform concentration of $c_{\text {ini }}=0.4$ and the temporal evolution of the concentration field. The red and blue colors indicate $c_{\alpha}$ and $c_{\beta}$ with high and low Li concentration, respectively (color figure online)

The diffusion problem, using (11) with chemical potential (55), is described by a fourth-order partial differential Eq. (4). The corresponding weak form is

$$
\int_{\mathcal{B}_{0}} \frac{\partial c}{\partial t} \delta c+m_{c} \nabla \mu \cdot \nabla(\delta c) \mathrm{d} V+\lambda \int_{\mathcal{B}_{0}} \Delta c \nabla m_{c} \cdot \nabla(\delta c)+m_{c} \Delta c \Delta(\delta c) \mathrm{d} V=0
$$

where $m_{c}=m_{i j}$ for all $i, j$; no-flux boundary conditions apply.

Finally, the weak form of the electrostatic problem becomes

$$
\int_{\mathcal{B}_{0}} \boldsymbol{D} \cdot \nabla(\delta \phi) \mathrm{d} V-\int_{\mathcal{B}_{0}} \rho_{e} \delta \phi \mathrm{d} V=0
$$

where the electric induction is specified in (56).

The unknown fields of the problems, $\chi, T, c$, and $\boldsymbol{D}$, are coupled through the constitutive relations. The resulting multi-physics problem is highly nonlinear and, to solve the corresponding numerical equations, it will be necessary to proceed with a suitable linearization of the constitutive relations. Linearization implies the definition of the hessian of the free energy that will include the fourth-order material tensor, the conductivity, the second-order electrical conductivity tensor, and others, as well as the corresponding coupling terms.

Because of the second-order derivatives in (61), the finite element approximation of the coupled problem requires the introduction of $C^{1}$ (at least) continuous piecewise-smooth approximation functions. Here, we use non-rational B-splines, which offer a high-order accuracy and robustness. For details of the implementation and demonstration of the efficiency, we refer to previous works $[4,5]$. The spline-based construction of the geometry requires to address some design issues and will be described in another paper.

\subsection{Purely elastic rod}

We start by analyzing the phase decomposition in an elastic cylindric specimen (rod) characterized by an initially uniform distribution of $\mathrm{Li}$ ions. The overall concentration is set initially to a constant value, $c(\boldsymbol{X}, 0)=$ 0.4 , and then let to evolve within a time interval of $2520 \mathrm{~s}$. Results are presented in terms of normalized time, i.e., $\bar{t}=t / \tau$ where $\tau=\ell^{2} / D$, see Table 2 .

The specimen has a length of $l=7 \mu \mathrm{m}$ and a radius of $r=0.5 \mu \mathrm{m}$, Fig. 8. The adopted mesh is composed by 189 elements with uniform size. The specimen is clamped at both ends, i. e., longitudinal displacements are constrained on the green planes in Fig. 8. The central points of the bases, indicated with yellow dots, are constrained in all directions. A no-flux condition is imposed on all boundaries through Lagrange multipliers [4].

Figure 8 shows a sequence of snapshots of the specimen at different times. The system immediately decomposes, exclusively by diffusion, into $\alpha$ and $\beta$-phases, whereby the specimen deforms as a consequence of swelling due to the intercalation $\mathrm{Li}$ ions. With time some regions grow at the expense of other ones and the phases coarsen. The material parameters chosen for the simulation lead to a symmetric equilibrium state characterized by two phases with concentration $c_{\alpha}=0.855$ and $c_{\beta}=0.145$, respectively. The application of an additional mechanical stress moves these values towards the mean concentration, as shown in Sect. 5. The expansion point for $J_{i}(c)$ is $c_{0}=0.25$.

Figure 9 shows the concentration distribution and the corresponding von Mises stress distribution at the time $\bar{t}=100$. High-concentration regions expand and are characterized by a low stress on the cylinder surface, 


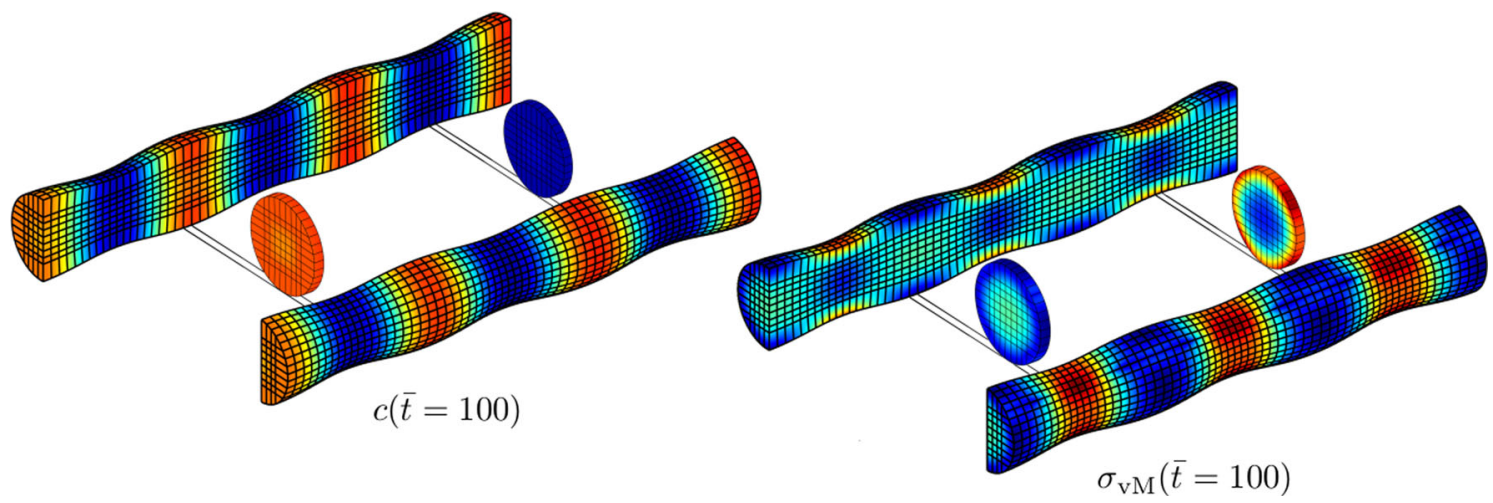

Fig. 9 Concentration field and equivalent von Mises stress in the clamped elastic rod at $\bar{t}=100$

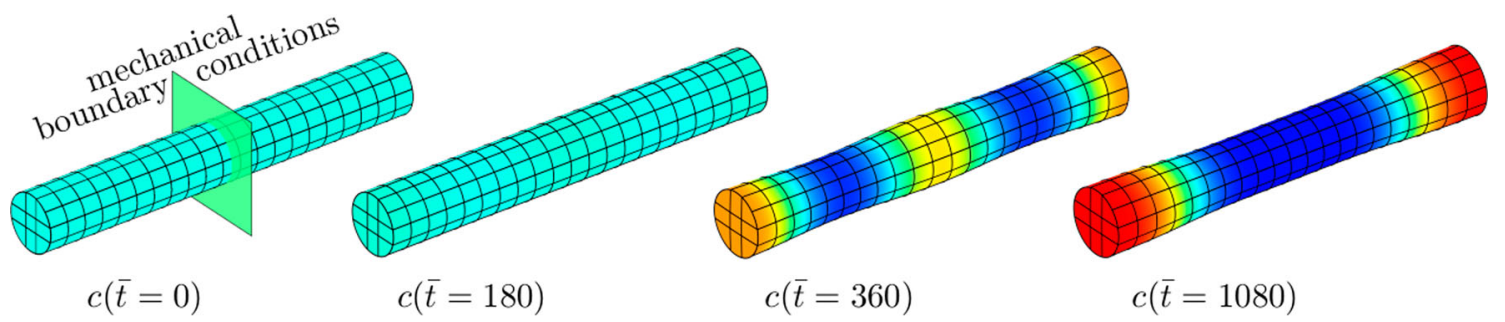

Fig. 10 Finite element model of the free elastic rod with an initially uniform concentration of $c=0.4$ and temporal evolution of the concentration field. The red and blue colors indicate $c_{\alpha}$ and $c_{\beta}$ with high and low Li concentration, respectively (color figure online)
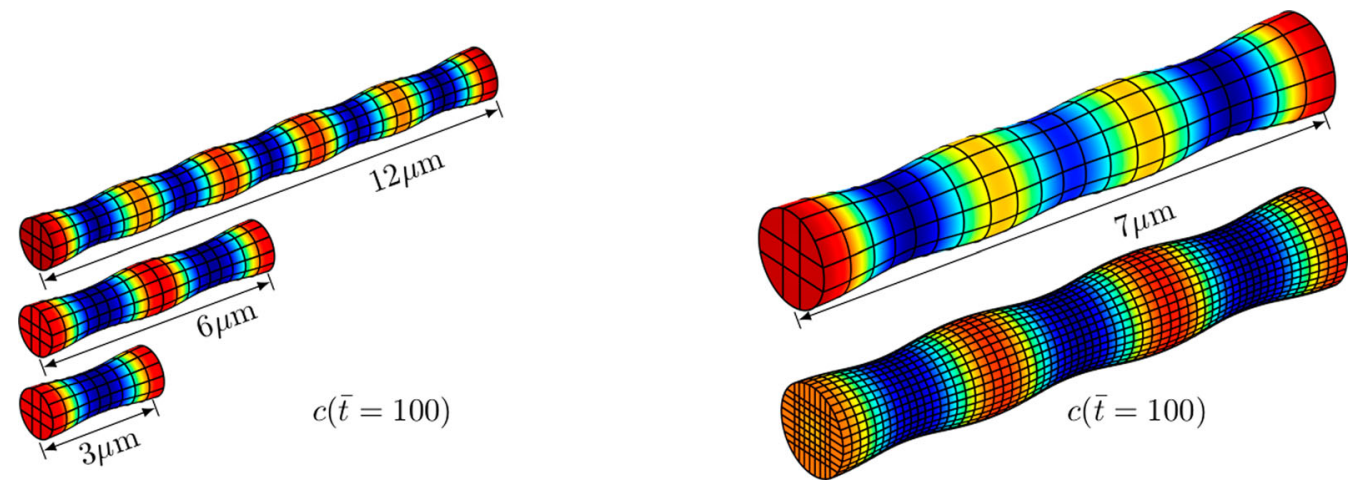

Fig. 11 Concentration distribution in clamped rods of different length at time $\bar{t}=100$ (left) and effects of two different meshes on the same rod Fig. 8 (right)

while low-concentration regions are characterized by a high compressive stress. The mutual dependence is less evident in the internal parts of the specimen, which reveal a more homogeneous stress.

Next, the phase decomposition problem is solved for a cylinder with unconstrained and load free bases, constrained only at the central section to exclude rigid body motion, see Fig. 10. For this free case, the interaction between diffusion and mechanical deformation is reduced to the presence of stresses related to intercalation. The specimen still shows phase coarsening and swelling, but these structures develop in much longer times. For example, at the time $\bar{t}=180$, the clamped specimen appears fully decomposed, whereas the free specimen is still in the initial configuration characterized by uniform concentration.

\subsubsection{Parametric analysis}

In order to assess the robustness of the proposed approach, we perform a parametric analysis considering only the geometry of the clamped specimen. 


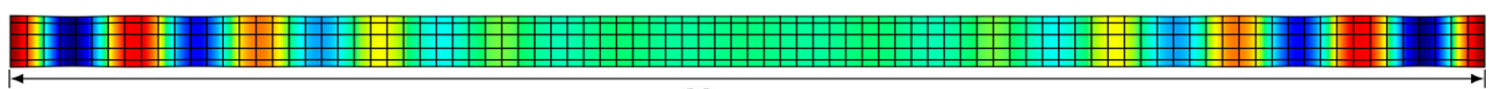

$$
30 \mu \mathrm{m}
$$

Fig. 12 Concentration field for a $30 \mu \mathrm{m}$ long clamped elastic rod at $\bar{t}=30$

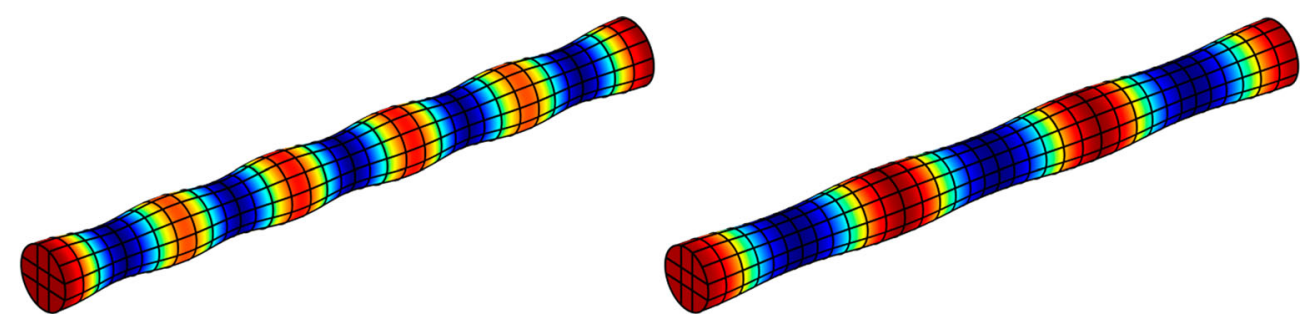

Fig. 13 Concentration fields for a $12 \mu \mathrm{m}$ long clamped elastic rod with gradient energy coefficient $\kappa$ (left) and $10 \kappa$ (right) shown at $\bar{t}=100$

Figure 11 compares the concentration fields at the time $\bar{t}=100$ for three specimens of length $l=3,6,12 \mu \mathrm{m}$, respectively. The shortest specimen results decomposed entirely at $\bar{t}=100$, whereas the concentration field in larger specimens is still evolving. The size of the evolving phases in the three specimens is similar but not equal. In particular, by measuring a single concentration phase as the distance between two half hills, the longest specimen develops five phases, whereas the medium specimen develops two phases, and the shortest develops only one phase. We can define the intrinsic wavelength of the system as approximately $2.4 \mu \mathrm{m}$ long. Note that, because of the driving surface energy, the $c_{\alpha}$-phases arrange initially at the system boundaries for $c_{\alpha}>c_{\beta}$ and $c \neq 0.5$; thus, all the specimens show the presence of high concentration phases at both clamped ends. For an additional $30 \mu \mathrm{m}$ long specimen, Fig. 12 shows an intermediate state of concentration decomposition.

Figure 11 (right) compares the concentration fields obtained with two different levels of mesh refinement, revealing a good agreement in terms of concentration phase location. Interestingly, further mesh refinements do not improve the results of the fine mesh in Fig. 11 (right).

Figure 13 describes the dependence of the results on the coefficient $\kappa$, a gradient energy factor that enters the interface term $|\nabla c|^{2}$ in Eq. (39). Using the $12 \mu \mathrm{m}$ long clamped elastic specimen, results have been obtained for the reference value of $\kappa$ reported in Table 2 and for a 10 times larger value, i. e., $\kappa_{1}=10 \kappa$. In dimensionless form, the interface term can be written as $|\nabla c|^{2}=\ell^{-2}|\tilde{\nabla} c|^{2}$, where $\tilde{\nabla}$ is a dimensionless operator and $\ell$ is a characteristic length. Consequently, the scaling of $\kappa \mapsto \kappa_{1}$ can be seen as a scaling of $\ell \mapsto \ell_{1}$

$$
\kappa_{1}|\nabla c|^{2}=10 \kappa|\nabla c|^{2}=\kappa\left(\frac{\sqrt{10}}{\ell}\right)^{2}|\tilde{\nabla} c|^{2}=: \kappa \ell_{1}^{-2}|\nabla c|^{2} .
$$

It follows the transition from $\alpha$ - to $\beta$-phase requires a longer time and lead to the formation of a smaller number of phases. We remark that the characteristic length may enter the normalized time, but the scaling is not one-to-one because of the presence of chemo-elastic interaction.

It has been shown that besides the concentration gradient coefficients, strain gradient coefficients or related gradient expressions also play a stabilizing role favoring uniform concentrations [51,55].

\subsubsection{Elasticity models}

The comparison is extended to the two elasticity models of Sect. 4.2, namely the classical neo-Hookean model with energy density (32) and the simplified finite elasticity model with energy density (33). The corresponding stresses are given by (52) and (53), respectively.

The geometry of the specimens $\left(l=7 \mu \mathrm{m}, r=0.5 \mu \mathrm{m}, c_{\text {ini }}=0.4, c_{0}=0.25\right)$ and the discretization (189 elements) are similar to the ones adopted in the previous section, but the time necessary for phase decomposition is longer, since here the elastic parameters $K$ and $G$ are kept constant.

Figure 14 compares the time evolution of the radii of the specimen as a function of the position along the longitudinal axis for the two elasticity models. At both clamped ends, the radius shows an initial jump from 0.5 to $\approx 0.53 \mu$, describing at the interior a waving profile characterized by three phases. Subsequently, the three 


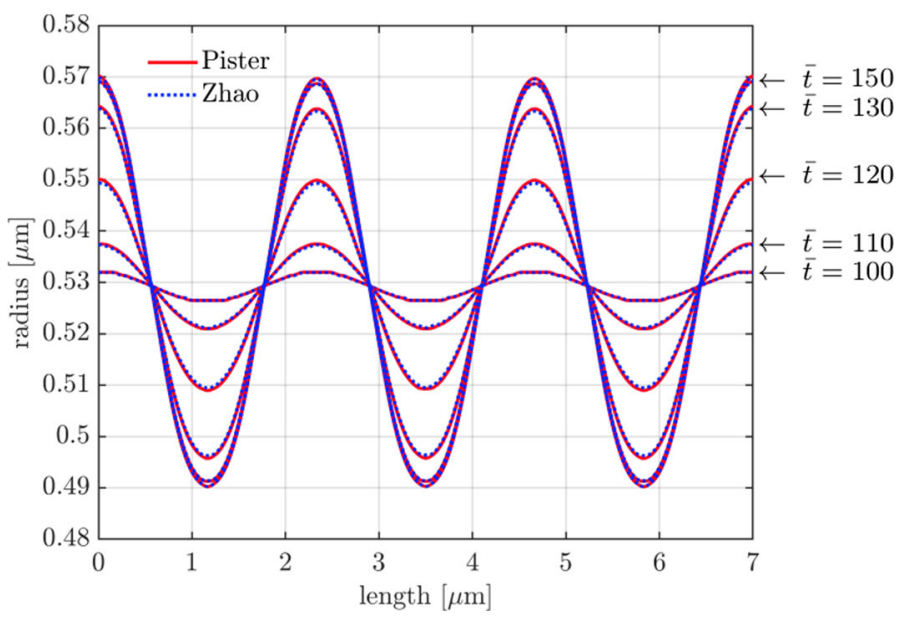

Fig. 14 Profiles of the rod radius along the longitudinal axis at different $\bar{t}$
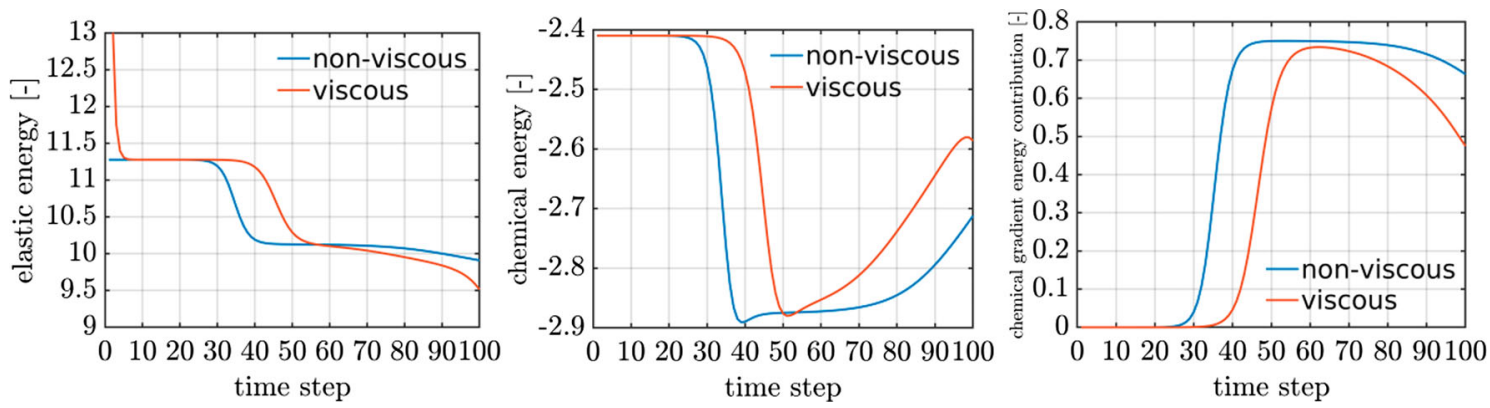

Fig. 15 Evolution of elastic, chemical and interface energy contributions with and without viscosity

phases evolve. The two elastic potentials result in a similar shape in all times, and the deviation between the two curves (blue and red lines in figure) is negligible. The computed average velocity for the entire swelling process is $10^{-11} \mathrm{~m} / \mathrm{s}$.

\subsection{Viscoelastic rod}

The effects of viscoelasticity are studied only for the $7 \mu \mathrm{m}$ long rod. To account for the viscous behavior, we adopt a one-parameter Maxwell model with $\tau_{1}=100 \ell^{2} / D, G_{1}=G_{0}$, keeping the other values according to the list in Table 2.

Figure 15 illustrates the time evolution of the elastic, chemical, and interface energy for the viscous model (red curves) and for the elastic model (blue curves) described in Sect. 6.2. The chemical energy plot reveals that phase decomposition is delayed in the viscous case; the minimum for the viscous line is attained at about 10 time steps later than for the elastic line. In later times, the kinetics of the reaction is accelerated as a consequence of the reduction of the elastic stresses.

Figure 16 shows snapshots the deformed configurations of the viscoelastic rod.

\subsection{Charging of a viscoelastic rod}

We conclude with an investigation on the charging process of a $7 \mu \mathrm{m}$ rod with initial concentration $c_{\text {ini }}=0.25$, and undergoing a constant normalized incoming charging Li flux of 0.064 at one of the basis, while the second basis is set as a no flux boundary. In order to display the results, we select ten points $P_{i}, i \in\{1, \ldots, 10\}$, located at the center of external finite elements, see Fig. 17. The point $P_{1}$ is close to the Li flux boundary, while the point $P_{10}$ is close to the no-flux boundary at the opposite end of the rod. 


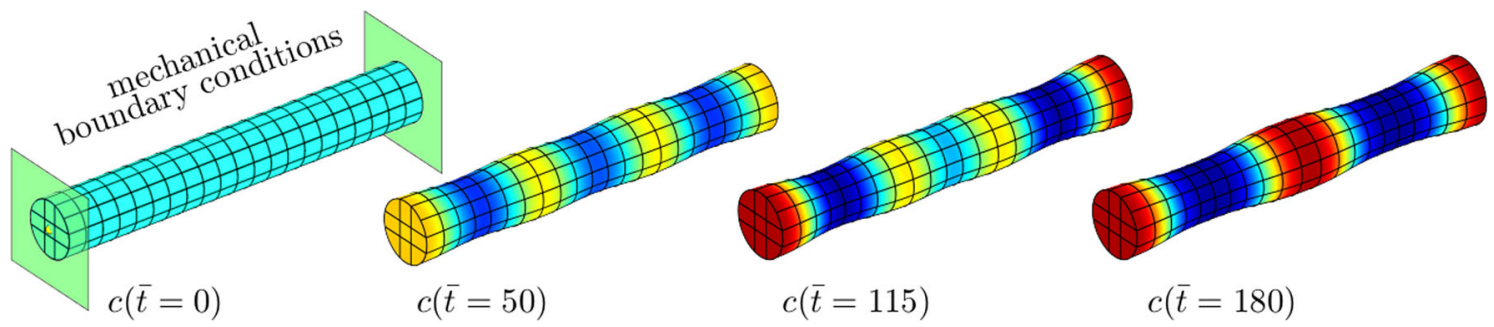

Fig. 16 Finite element model of the clamped viscoelastic rod with an initially uniform concentration of $c_{\text {ini }}=0.4$ and evolution of the concentration field. The red and blue colors indicate $c_{\alpha}$ and $c_{\beta}$ with high and low Li concentration, respectively (color figure online)
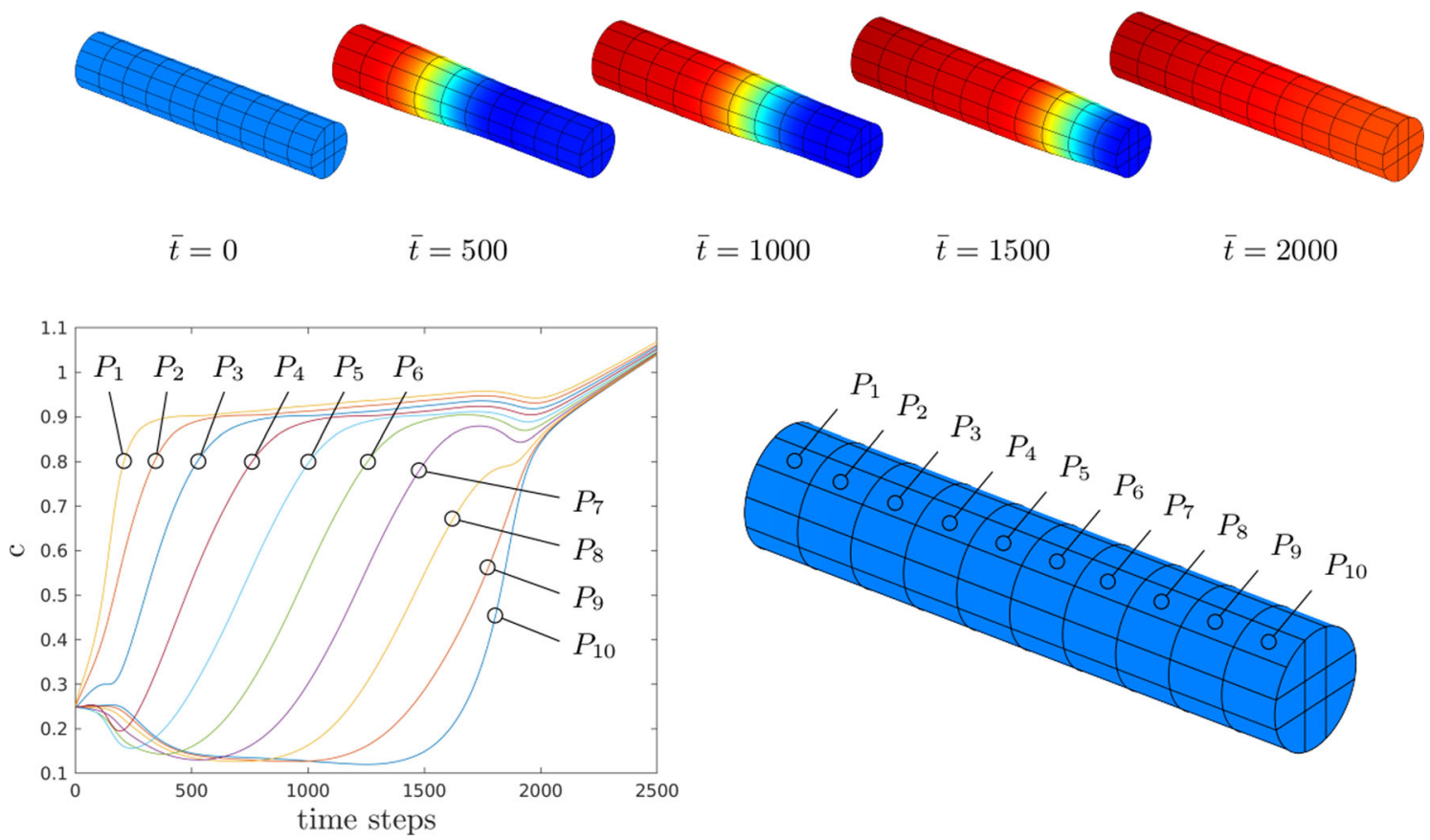

Fig. 17 Evolution of concentration in a rod during charging

The dynamics of charging is shown in the plots of Fig. 17. At the point $P_{1}$, the concentration increases until it reaches the equilibrium concentration $c_{\alpha}$. In contrast, at the point $P_{10}$, energy minimization leads to a local concentration $c_{\beta}$. The highest concentration values are observed at the charging side and decrease progressively with the distance from it, i. e., $c\left(P_{i}\right)>c\left(P_{j}\right), j>i$. Due to the constant incoming flux, the charging process is characterized by an almost constant velocity $\partial_{t} c\left(P_{i}\right)$ at intermediate times between the initial and final equilibrium concentrations. Plots show that, within the first 2300 time steps before full charging, concentrations remain in the interval $[0,1]$. It bears emphasis that in real charging processes, the incoming $\mathrm{Li}$ flux is not constant but reduces with raising concentration. An analogous effect is induced by viscoelasticity.

Clearly, because of dissipative phenomena, the relevance of viscosity or inelasticity comes heavily into play when charging and discharging cycles are considered at the anode, calling for more sophisticated simulations that will be conducted in future studies.

\section{Conclusions}

We derived a multi-field model for lithium electrodes during charging and discharging. Ion flux, diffusion and phase decomposition, temperature, electricity, ion intercalation, and swelling contribute to the material internal energy and to the development of large elastic and inelastic mechanical deformations. Coupling between concentration, electricity, temperature, and displacement fields arises from the constitutive relations 
and is accounted for in the kinematics through the multiplicative decomposition of the deformation gradient, which splits into elastic and inelastic parts. A Taylor series expansion is used to approximate the unknown inelastic deformation due to ion intercalation. We show that the reference concentration $c_{0}$ strongly influences the decomposition behavior.

Our model is consistently derived from the basic laws of thermodynamics and reflects the fundamental aspects of the anode charging process. We have illustrated the properties of the proposed model through several examples and have pointed out that chemomechanical interaction affects the equilibrium concentrations of the phases. For typical charging processes, the electromechanical coupling remains marginal, and the model compares - for special cases - to previous electrode charging models available in the literature.

We observed that two neo-Hookean models of finite elasticity lead to similar evolutions of the concentration distribution, i. e., they deliver the same intrinsic wavelength during the coupled decomposition. A Maxwelltype viscoelastic material model reveals that a viscoelastic specimens delays the onset of phase decomposition, but the decomposition accelerates at later stages.

The finite element simulations here described demonstrate the robustness of the proposed electrochemicalthermomechanical model.

Acknowledgements The authors gratefully acknowledge the support of the Deutsche Forschungsgemeinschaft (DFG) under the Project WE-2525/8-1 and WE-2525/11-1.

Funding Open Access funding enabled and organized by Projekt DEAL.

Open Access This article is licensed under a Creative Commons Attribution 4.0 International License, which permits use, sharing, adaptation, distribution and reproduction in any medium or format, as long as you give appropriate credit to the original author(s) and the source, provide a link to the Creative Commons licence, and indicate if changes were made. The images or other third party material in this article are included in the article's Creative Commons licence, unless indicated otherwise in a credit line to the material. If material is not included in the article's Creative Commons licence and your intended use is not permitted by statutory regulation or exceeds the permitted use, you will need to obtain permission directly from the copyright holder. To view a copy of this licence, visit http://creativecommons.org/licenses/by/4.0/.

Compliance with ethical standards

Conflict of interest The authors declare that they have no conflict of interest.

\section{Appendix}

Material and spatial configuration

For the transformation from the spatial into the material configuration and vice versa, the usual transformation rules hold. The push-forward of the (covariant) material gradient $\boldsymbol{E}(\boldsymbol{x}, t)=\nabla_{\boldsymbol{X}} \phi$ into its spatial counterpart $\boldsymbol{e}(\boldsymbol{x}, t)=\nabla_{x} \phi$ is given by

$$
\boldsymbol{e}=\nabla_{\boldsymbol{x}} \phi=\left(\nabla_{\boldsymbol{X}}^{\top} \phi \boldsymbol{F}^{-1}\right)^{\top}=\boldsymbol{F}^{-\top} \nabla_{\boldsymbol{X}} \phi=\boldsymbol{F}^{-\top} \boldsymbol{E},
$$

and the pull-back reads $\boldsymbol{E}=\boldsymbol{F}^{\top} \boldsymbol{e}$. The transformation of oriented surface elements d $\boldsymbol{s}$ in spatial and $\mathrm{d} \boldsymbol{S}$ in material description is governed by the Nanson'rule,

$$
\mathrm{d} \boldsymbol{s}=\boldsymbol{n} \mathrm{d} s=J \boldsymbol{F}^{-\top} \boldsymbol{N} \mathrm{d} S=J \mathbf{F}^{-\top} \mathrm{d} \boldsymbol{S},
$$

where $N$ is the outward unit normal on the boundary $\partial \mathcal{B}_{0}$ of the body in its material configuration and $\boldsymbol{n}$ is unit normal of the current boundary $\partial \mathcal{B}_{t}$.

\section{Theorems}

Here, we briefly provide three important theorems for a scalar and vectorized quantities $\bullet$ and $\hat{\bullet}$, respectively, which will be used subsequently:

$$
\int_{\mathcal{B}^{*}} \bullet \mathrm{d} v=0 \quad \forall \mathcal{B}^{*} \subset \mathcal{B} \Leftrightarrow \bullet=0 \quad \forall x \in \mathcal{B} \quad \text { (Localization theorem) }
$$


Table 3 Nomenclature and SI units for used quantities

\begin{tabular}{llllll}
\hline$\rho, \rho_{0}$ & Mass density & $\left(\mathrm{kg} / \mathrm{m}^{3}\right)$ & $\boldsymbol{b}, \boldsymbol{B}$ & Body force density & $\left(\mathrm{kg} / \mathrm{m}^{2} \mathrm{~s}^{2}\right)$ \\
$h$ & Mass flux & $\left(\mathrm{kg} / \mathrm{sm}^{3}\right)$ & $\boldsymbol{v}$ & Velocity & $(\mathrm{m} / \mathrm{s})$ \\
$c_{k}$ & Concentration & $(-)$ & $\boldsymbol{d}, \boldsymbol{D}$ & Electrical induction & $\left(\mathrm{As} / \mathrm{m}^{2}\right)$ \\
$\boldsymbol{j}$ & Mass flux vector & $\left(\mathrm{kg} / \mathrm{m}^{2}\right)$ & $z$ & Charge per unit mass & $(\mathrm{As} / \mathrm{kg})$ \\
$\nu_{k j}$ & Stoichiometric coeff. & $(-)$ & $T$ & Temperature & $(\mathrm{K})$ \\
$\mathcal{J}_{j}$ & Reaction rate & $\left(\mathrm{kg} / \mathrm{sm}^{3}\right)$ & $C_{T}$ & Specific heat capacity & $\left.(\mathrm{kg} / \mathrm{Kms})^{2}\right)$ \\
$\boldsymbol{\sigma}, \boldsymbol{P}$ & Stress & $\left(\mathrm{kg} / \mathrm{ms}^{2}\right)$ & $\mathcal{R}$ & Rate of heat supply & $\left(\mathrm{kg} / \mathrm{ms}^{3}\right)$ \\
$\boldsymbol{t}$ & Traction & $\left(\mathrm{kg} / \mathrm{ms}^{2}\right)$ & $\boldsymbol{h}_{T} \boldsymbol{H}_{T}$ & Thermal flux vector & $\left(\mathrm{kg} / \mathrm{s}^{3}\right)$ \\
\hline
\end{tabular}

$$
\begin{aligned}
& \int_{\mathcal{B}} \nabla \cdot \hat{\bullet} \mathrm{d} v=\int_{\partial \mathcal{B}} \hat{\bullet} \cdot \boldsymbol{n} \mathrm{d} s \quad \text { (Divergence theorem) } \\
& \frac{\mathrm{d}}{\mathrm{d} t} \int_{\mathcal{B}} \bullet \mathrm{d} v=\frac{\mathrm{d}}{\mathrm{d} t} \int_{\mathcal{B}} \bullet J \mathrm{~d} V=\int_{\mathcal{B}}\left[\bullet+\frac{j}{J} \bullet\right] \mathrm{d} v \quad \text { (Reynold's transport) }
\end{aligned}
$$

where $\mathcal{B}^{*}$ are subdomains contained in $\mathcal{B}$. Additionally, common definitions are

$$
\begin{aligned}
\bullet & \equiv \frac{\mathrm{d} \bullet}{\mathrm{d} t}=\frac{\partial \bullet}{\partial t}+\boldsymbol{v} \cdot \nabla \bullet \quad \forall \boldsymbol{x} \in \mathcal{B} \quad \text { (substantial time derivative) } \\
\delta_{c} & \equiv \frac{\delta \bullet}{\delta c}=\frac{\partial \bullet}{\partial c}-\nabla \cdot \frac{\partial \bullet}{\partial \nabla c} \quad \forall \boldsymbol{x} \in \mathcal{B} \quad \text { (variational derivative) }
\end{aligned}
$$

where $\boldsymbol{v}(\boldsymbol{x}, t)$ is the flow velocity.

\section{References}

1. Aifantis, K.E., Hackney, S.A., Kumar, R.V.: High Energy Density Lithium Batteries: Materials, Engineering, Applications. Wiley, Hoboken (2010)

2. Anand, L.: A Cahn-Hilliard-type theory for species diffusion coupled with large elastic-plastic deformations. J. Mech. Phys. Solids 60(12), 1983-2002 (2012)

3. Anders, D., Weinberg, K.: A variational approach to the decomposition of unstable viscous fluids and its consistent numerical approximation. ZAMM J. Appl. Math. Mech. Z. Angew. Math. Mech. 91(8), 609-629 (2011)

4. Anders, D., Hesch, C., Weinberg, K.: Computational modeling of phase separation and coarsening in solder alloys. Int. J. Solids Struct. 49(13), 1557-1572 (2012)

5. Anders, D., Weinberg, K.: Simulation of diffusion induced phase separation and coarsening in binary alloys. Comput. Mater. Sci. 50(4), 1359-1364 (2011)

6. Areias, P., Matouš, K.: Finite element formulation for modeling nonlinear viscoelastic elastomers. Comput. Methods Appl. Mech. Eng. 197(51), 4702-4717 (2008)

7. Ashuri, M., He, Q., Shaw, L.L.: Silicon as a potential anode material for li-ion batteries: where size, geometry and structure matter. Nanoscale 8, 74-103 (2016)

8. Bazant, M.Z.: Theory of chemical kinetics and charge transfer based on nonequilibrium thermodynamics. Acc. Chem. Res. 46(5), 1144-1160 (2013)

9. Beaulieu, L.Y., Beattie, S.D., Hatchard, T.D., Dahn, J.R.: The electrochemical reaction of lithium with tin studied by in situ AFM. J. Electrochem. Soc. 150(4), A419 (2003)

10. Berla, L.A., Lee, S.W., Ryu, I., Cui, Y., Nix, W.D.: Robustness of amorphous silicon during the initial lithiation/delithiation cycle. J. Power Sources 258, 253-259 (2014)

11. Bonet, J., Gil, A.J., Ortigosa, R.: A computational framework for polyconvex large strain elasticity. Comput. Methods Appl. Mech. Eng. 283, 1061-1094 (2015)

12. Coleman, B.D., Noll, W.: Material symmetry and thermostatic inequalities in finite elastic deformations. Arch. Ration. Mech. Anal. 15, 87-111 (1964)

13. Dal, H., Miehe, C.: Computational electro-chemo-mechanics of lithium-ion battery electrodes at finite strains. Comput. Mech. 55, 303-325 (2015)

14. de Groot, S.R., Mazur, P.: Non-equilibrium Thermodynamics. North-Holland, Amsterdam (1962)

15. de Jongh, P.E., Notten, P.H.L.: Effect of current pulses on lithium intercalation batteries. Solid State Ionics 148(3), 259-268 (2002)

16. Eckart, C.: The thermodynamics of irreversible processes. IV. The theory of elasticity and anelasticity. Phys. Rev. 73, 373-382 (1948)

17. Ferguson, T.R., Bazant, M.Z.: Nonequilibrium thermodynamics of porous electrodes. J. Electrochem. Soc. 159(12), A1967A1985 (2012)

18. Goddard, J.D.: Dissipation potentials for reaction-diffusion systems. Ind. Eng. Chem. Res. 54(16), 4078-4083 (2015)

19. Gregersen, M.M., Okkels, F., Bazant, M.Z., Bruus, H.: Topology and shape optimization of induced-charge electro-osmotic micropumps. New J. Phys. 11, 075019 (2009) 
20. Hesch, C., Schuß, S., Dittmann, M., Franke, M., Weinberg, K.: Isogeometric analysis and hierarchical refinement for higherorder phase-field models. Comput. Methods Appl. Mech. Eng. 303, 185-207 (2016)

21. Hu, B., Ma, Z., Lei, W., Zou, Y., Lu, C.: A chemo-mechanical model coupled with thermal effect on the hollow core-shell electrodes in lithium-ion batteries. Theor. Appl. Mech. Lett. 7(4), 199-206 (2017)

22. Huttin, M., Kamlah, M.: Phase-field modeling of stress generation in electrode particles of lithium ion batteries. Appl. Phys. Lett. 101(13), $133902(2012)$

23. Kim, H., Chou, C.-Y., Ekerdt, J.G., Hwang, G.S.: Structure and properties of Li-Si alloys: a first-principles study. J. Phys. Chem. C 115(5), 2514-2521 (2011)

24. Lee, E.H.: Elastic-plastic deformation at finite strain. J. Appl. Mech. 36, 1-6 (1969)

25. Lee, S., Park, J., Sastry, A.M., Lu, W.: Molecular dynamics simulations of SOC-dependent elasticity of LixMn2O4 spinels in Li-ion batteries. J. Electrochem. Soc. 160(6), a968-a972 (2013)

26. Di Leo, C.V., Rejovitzky, E., Anand, L.: A cahn-hilliard-type phase-field theory for species diffusion coupled with large elastic deformations: application to phase-separating li-ion electrode materials. J. Mech. Phys. Solids 70, 1-29 (2014)

27. Li, K., Xie, H., Liu, J., Ma, Z., Zhou, Y., Xue, D.: From chemistry to mechanics: bulk modulus evolution of Li-Si and Li-Sn alloys via the metallic electronegativity scale. Phys. Chem. Chem. Phys. 15, 17658-17663 (2013)

28. Ma, Z., Wu, H., Wang, Y., Pan, Y., Lu, C.: An electrochemical-irradiated plasticity model for metallic electrodes in lithium-ion batteries. Int. J. Plast 88, 188-203 (2017)

29. Malik, R., Burch, D., Bazant, M., Ceder, G.: Particle size dependence of the ionic diffusivity. Nano Lett. 10(10), 4123-4127 (2010)

30. Natsiavas, P.P., Weinberg, K., Rosato, D., Ortiz, M.: Effect of prestress on the stability of electrode-electrolyte interfaces during charging in lithium batteries. J. Mech. Phys. Solids 95, 92-111 (2016)

31. Nauman, E.B., He, D.Q.: Nonlinear diffusion and phase separation. Chem. Eng. Sci. 56(6), 1999-2018 (2001)

32. Nitta, N., Yushin, G.: High-capacity anode materials for lithium-ion batteries: choice of elements and structures for active particles. Part. Part. Syst. Charact. 31(3), 317-336 (2014)

33. O’Connell, J.P., Haile, J.M.: Thermodynamics: Fundamentals for Applications. Cambridge University Press, Cambridge (2005)

34. Ortiz, M., Stainier, L.: The variational formulation of viscoplastic constitutive updates. Comput. Methods Appl. Mech. Eng. 171(3), 419-444 (1999)

35. Pandolfi, A., Gizzi, A., Vasta, M.: Visco-electro-elastic models of fiber-distributed active tissues. Meccanica 52, 3399-3415 (2017)

36. Peiyu, H., Geng, C., Jian, G., Yantao, Z., Lianqi, Z.: Li-ion batteries: phase transition. Chin. Phys. B 25(1), 016104 (2016)

37. Qi, Y., Hector, L.G., James, C., Kim, K.J.: Lithium concentration dependent elastic properties of battery electrode materials from first principles calculations. J. Electrochem. Soc. 161(11), F3010-F3018 (2014)

38. Rejovitzky, E., Di Leo, C.V., Anand, L.: A theory and a simulation capability for the growth of a solid electrolyte interphase layer at an anode particle in a li-ion battery. J. Mech. Phys. Solids 78, 210-230 (2015)

39. Sauer, R.A., Ghaffari, R., Gupta, A.: The multiplicative deformation split for shells with application to growth, chemical swelling, thermoelasticity, viscoelasticity and elastoplasticity. Int. J. Solids Struct. 174-175, 53-68 (2019)

40. Schuß, S., Weinberg, K., Hesch, C.: Thermomigration in snpb solders: material model. Mech. Mater. 121, 31-49 (2018)

41. Sethuraman, V.A., Chon, M.J., Shimshak, M., Van Winkle, N., Guduru, P.R.: In situ measurement of biaxial modulus of si anode for li-ion batteries. Electrochem. Commun. 12(11), 1614-1617 (2010)

42. Shenoy, V.B., Johari, P., Qi, Y.: Elastic softening of amorphous and crystalline Li-Si phases with increasing li concentration: a first-principles study. J. Power Sources 195(19), 6825-6830 (2010)

43. Sidoroff, F.: Un modele viscoelastique non lineaire avec configuration intermediaire. J. de Mech 13, 679-713 (1974)

44. Simo, J.C., Ortiz, M.: A unified approach to finite deformation elastoplastic analysis based on the use of hyperelastic constitutive equations. Comput. Methods Appl. Mech. Eng. 49(2), 221-245 (1985)

45. Simo, J.C., Pister, K.S.: Remarks on rate constitutive equations for finite deformation problems: computational implications. Comput. Methods Appl. Mech. Eng. 46(2), 201-215 (1984)

46. Simo, J.C., Taylor, R.L.: Penalty function formulations for incompressible nonlinear elastostatics. Comput. Methods Appl. Mech. Eng. 35(1), 107-118 (1982)

47. Stein, P., Zhao, Y., Xu, B.-X.: Electrochemical reactions in li-ion battery electrodes and their interaction with mechanical stresses: size effects, phase segregation, and crack propagation. Meet. Abstr. MA2017-01(1), 133 (2017)

48. Stojanović, R., Djurić, S., Vujošević, L.: On finite thermal deformations. Arch. Mech. Stosow. 1, 103-108 (1964)

49. Triani, V., Papenfuss, C., Cimmelli, V.A., Muschik, W.: Exploitation of the second law: Coleman-Noll and Liu procedure in comparison. J. Nonequilib. Thermodyn. 33(1), 47-60 (2008)

50. Truesdell, C., Toupin, R.A.: The classical field theories. In: Flügge, S. (ed.) Handbuch der Physik, Volume III/1. Springer, Berlin (1960)

51. Tsagrakis, I., Aifantis, E.C.: Thermodynamic coupling between gradient elasticity and a Cahn-Hilliard type of diffusion: size-dependent spinodal gaps. Continuum Mech. Thermodyn. 29(6), 1181-1194 (2017)

52. Tschoegl, N.W.: The Phenomenological Theory of Linear Viscoelastic Behavior: An Introduction. Springer, Berlin (1989)

53. Tsiropoulos, I., Tarvydas, D., Lebedeva, N.: Li-Ion Batteries for Mobility and stationary Storage Applications-Scenarios for Costs and Market Growth, eur 29440 en. Technical Report, Publications Office of the European Union (2018)

54. Weinberg, K., Pandolfi, A.: A material model for electroactive polymers. In: Naumenko, K., Assmus, M. (eds.) Advanced Methods of Continuum Mechanics for Materials and Structures, Advanced Structured Materials, pp. 119-134. Springer, Singapore (2016)

55. Weinberg, K., Hesch, C.: A high-order finite deformation phase-field approach to fracture. Continuum Mech. Thermodyn. 29, 935-945 (2017)

56. Weinberg, K., Werner, M., Anders, D.: A chemo-mechanical model of diffusion in reactive systems. Entropy 20(2), 140 (2018) 
57. Werner, M., Weinberg, K.: Coupled Thermal and Electrochemical Diffusion in Solid State Battery Systems, pp. 519-535. Springer, Cham (2019)

58. Yabuuchi, N., Kubota, K., Aoki, Y., Komaba, S.: Understanding particle-size-dependent electrochemical properties of Li2Mno3-based positive electrode materials for rechargeable lithium batteries. J. Phys. Chem. C 120(2), 875-885 (2016)

59. Yang, Q., Stainier, L., Ortiz, M.: A variational formulation of the coupled thermo-mechanical boundary-value problem for general dissipative solids. J. Mech. Phys. Solids 54(2), 401-424 (2006)

60. Yao, Y., McDowell, M., Ryu, I., Wu, H., Liu, N., Hu, L., Nix, W.D., Cui, Y.: Interconnected silicon hollow nanospheres for lithium-ion battery anodes with long cycle life. Nano Lett. 11, 2949-2954 (2011)

61. Zhang, S.S.: The effect of the charging protocol on the cycle life of a li-ion battery. J. Power Sources 161(2), 1385-1391 (2006)

62. Zhang, T., Kamlah, M.: A nonlocal species concentration theory for diffusion and phase changes in electrode particles of lithium ion batteries. Continuum Mech. Thermodyn. 30(3), 553-572 (2018)

63. Zhang, X., Wang, Q.J., Shen, H.: A multi-field coupled mechanical-electric-magnetic-chemical-thermal (memct) theory for material systems. Comput. Methods Appl. Mech. Eng. 341, 133-162 (2018)

64. Zhang, X.-Y., Song, W.-L., Liu, Z., Chen, H.-S., Li, T., Wei, Y., Fang, D.-N.: Geometric design of micron-sized crystalline silicon anodes through in situ observation of deformation and fracture behaviors. J. Mater. Chem. A 5, 12793-12802 (2017)

65. Zhao, Y., Stein, P., Bai, Y., Al-Siraj, M., Yang, Y., Xu, B.-X.: A review on modeling of electro-chemo-mechanics in lithium-ion batteries. J. Power Sources 413, 259-283 (2019)

66. Zhao, Y., Stein, P., Xu, B.-X.: Isogeometric analysis of mechanically coupled Cahn-Hilliard phase segregation in hyperelastic electrodes of li-ion batteries. Comput. Methods Appl. Mech. Eng. 297, 325-347 (2015)

67. Zhao, Y., Xu, B.-X., Stein, P., Gross, D.: Phase-field study of electrochemical reactions at exterior and interior interfaces in li-ion battery electrode particles. Comput. Methods Appl. Mech. Eng. 312, 428-446 (2016)

Publisher's Note Springer Nature remains neutral with regard to jurisdictional claims in published maps and institutional affiliations. 